\title{
Theory of driven Higgs oscillations and third-harmonic generation in unconventional superconductors
}

\author{
Lukas Schwarz 10 and Dirk Manske \\ Max Planck Institute for Solid State Research, 70569 Stuttgart, Germany
}

(Received 25 November 2019; accepted 13 April 2020; published 21 May 2020)

\begin{abstract}
Higgs spectroscopy is a new field in which Higgs modes in nonequilibrium superconductors are analyzed to gain information about the ground state. One experimental setup in which the Higgs mode in $s$-wave superconductors was observed is periodic driving with terahertz $(\mathrm{THz})$ light, which shows resonances in the third-harmonic generation (THG) signal if twice the driving frequency matches the energy of the Higgs mode. We derive expressions of the driven gap oscillations for arbitrary gap symmetry and calculate the THG response. We demonstrate that the possible Higgs modes for superconductors with nontrivial gap symmetry can lead to additional resonances if twice the driving frequency matches the energy of these Higgs modes and we disentangle the influence of charge density fluctuations (CDF) to the THG signal within our clean-limit analysis. With this we show that THG experiments on unconventional superconductors allow for detection of their Higgs modes. This paves the way for future studies on realistic systems including additional features to understand the collective excitation spectra of unconventional superconductors.
\end{abstract}

DOI: 10.1103/PhysRevB.101.184519

\section{INTRODUCTION}

In recent years with the emergence of terahertz $(\mathrm{THz})$ spectroscopy, studies on matter were possible in regimes which were inaccessible before [1-3]. Due to the energy of $\mathrm{THz}$ radiation in the $\mathrm{meV}$ range, gentle excitations of materials can be performed without destroying the quantum coherence of the whole system. This allows for controlled experiments in nonequilibrium situations from which ground-state properties can be uncovered.

One interesting field within the $\mathrm{THz}$ studies are excitations of collective modes like the Higgs mode in superconductors [4-9]. In equilibrium this mode arises due to the spontaneous $\mathrm{U}(1)$ symmetry breaking in the superconducting state which is characterized by a Mexican hat-shaped free energy potential (Fig. 1). In principle, there are two different collective modes possible: a phase excitation azimuthally around the brim of the Mexican hat - the Goldstone mode and an amplitude excitation radially - the Higgs mode. However, due to the Anderson-Higgs mechanism [10], the originally massless Goldstone mode gets shifted to the plasma energy, whereas the Higgs mode remains with an energy of $2 \Delta$, i.e., the energy of the superconducting gap [5].

The Higgs mode does not possess a dipole moment therefore it does not couple linearly to light. This makes its experimental excitation and detection difficult. Except in the

Published by the American Physical Society under the terms of the Creative Commons Attribution 4.0 International license. Further distribution of this work must maintain attribution to the author(s) and the published article's title, journal citation, and DOI. Open access publication funded by the Max Planck Society. special situation, where a charge-density wave order coexist with the superconducting order on a similar energy range providing a coupling between the two orders and making the Higgs mode Raman-active [11-16], more effort has to be expended for an observation of the Higgs mode. Only recently with ultrafast $\mathrm{THz}$ laser technology an excitation and observation of the Higgs mode in a pump-probe experiment for the $s$-wave system $\mathrm{NbN}$ and $\mathrm{Nb}_{1-x} \mathrm{Ti}_{x} \mathrm{~N}$ was possible $[17,18]$ and first experiments on cuprate $d$-wave systems were reported $[19,20]$. In these experiments, oscillations of the optical conductivity after a short $\mathrm{THz}$ pulse resulting from Higgs oscillations in nonequilibrium could be observed. Alternatively, it was shown theoretically that time-resolved ARPES experiments could be be used in a pump-probe setup as well for the observation of Higgs oscillations [21-23]. These oscillations in nonequilibrium were predicted already before the experiment [24-32] and lead to a great variety of further research considering coupling to phonons [33,34], quasi-1d systems [35], multiband superconductors with additional Leggett modes [36,37], strongly coupled regimes beyond BCS theory [38], current-carrying states [39,40], and condensates with $d$-wave symmetry [41,42].

Especially the consideration of unconventional superconductors may open a new field of spectroscopy. For superconductors with nontrivial gap symmetry, there are multiple Higgs modes possible which can be decomposed into the irreducible representation of the underlying lattice symmetry [7] and can be understood as asymmetric oscillations of the condensate. Therefore one may infer the symmetry of the gap from a careful analysis of the Higgs modes in a chosen system [42].

Recent studies on light-induced superconductivity in different systems [43-48] raise the question on how to define superconductivity in a short-lived nonequilibrium state. One 


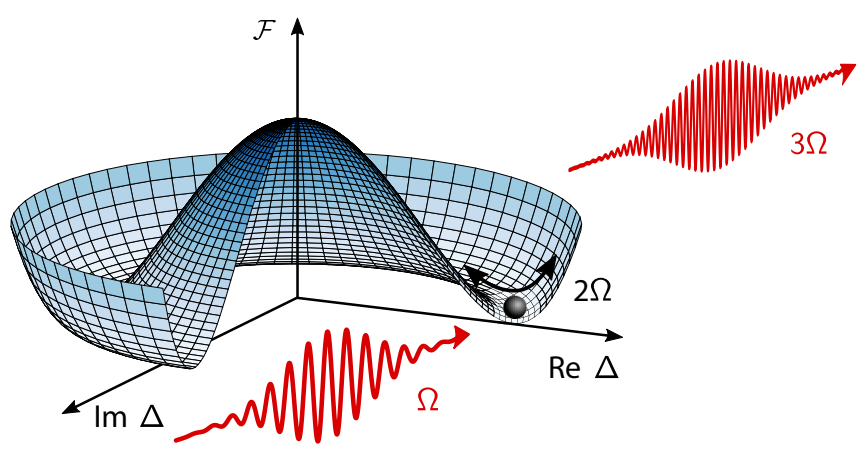

FIG. 1. Free energy $\mathcal{F}$ of a superconductor as function of the complex order parameter $\Delta$ shaped as a Mexican hat. Radial oscillations in the potential correspond to amplitude (Higgs) oscillations of the order parameter. If the superconductor is driven by light of frequency $\Omega$, enforced Higgs oscillations occur with a frequency of $2 \Omega$ due to the quadratic coupling of light. The system responds with third-harmonic generation (THG) $3 \Omega$, resulting from the $\Omega$ drive plus $2 \Omega$ Higgs oscillations.

criteria for superconductivity is the Meissner effect, which is induced on a microscopic level by the Anderson-Higgs mechanism. Hence, a measurement of the Higgs mode is an equivalent probe and it is therefore important to understand Higgs oscillations for all kind of unconventional superconductors.

As pump-probe experiments are difficult to conduct due to the requirement of ultrashort single-cycle $\mathrm{THz}$ pulses within the energy range of the superconducting gap, it is worth to consider alternative experimental setups for measuring Higgs oscillations. Instead of quenching the superconducting condensate with a $\mathrm{THz}$ pump pulse and observing the intrinsic Higgs oscillations directly, a periodic driving scheme with multicycle pulses can be used (Fig. 1). The nonlinear coupling to the condensate of light $A(t)$ with frequency $\Omega$ induces oscillations of the energy gap $\delta \Delta(t)$ with twice the driving frequency $2 \Omega$. This nonlinear coupling also induces higher-order currents $j$, where a third-harmonic generation (THG) component $j^{(3)}(t)$ arises, resulting from the driven gap oscillations, i.e., $j^{(3)}(t) \propto A(t) \delta \Delta(t)$ [49]. If the effective $2 \Omega$ driving frequency is tuned to the energy of the Higgs mode $2 \Delta$, a resonance occur in the gap oscillation and consequently also in the THG intensity, which indicates the existence of the Higgs mode. This effect can be measured in the transmitted electric field.

This setup was already successfully demonstrated in an experiment for the $s$-wave superconductor $\mathrm{NbN}$ [50,51]. More recently, experiments on several different cuprates were performed, where a possible new mode at an energy below the symmetric $2 \Delta$ Higgs mode was observed in the THG spectrum [52]. This experiment and further ongoing efforts in performing these kinds of experiments on unconventional superconductors require an understanding of the underlying physics. Hence, this work investigates the THG response for different unconventional superconductors to study the influence of gap symmetries.

There are several experimental difficulties while performing THG experiments. With current technology, the frequency of the driving field in the $\mathrm{THz}$ range cannot be tuned arbitrarily. The experiment is performed in such a way that the driving frequency is fixed and the temperature is changed to obtain the resonance condition.

Second and more seriously is the fact that the Higgs mode is not the only process leading to a resonance in the THG spectrum. Due to the driving, charge density fluctuations (CDF) are excited as well which resonate at the pair-breaking energy $2 \Delta$ coinciding with the Higgs mode. Depending on the system considered, these contributions may exceed the Higgs contribution by several orders [53]; however, an analysis beyond BCS approximation shows that the situation may reverse again [54]. Studying the polarization dependence of the THG signal can help to distinguish between the contributions, which is however not as clear as to be wished as the polarization dependence depends strongly on the dispersion and pairing interaction [55]. However, other papers pointed out that for superconductors in the dirty-limit the paramagnetic coupling to light plays a crucial role which leads to a dominant Higgs contribution in the THG signal [56-60]. This shows that a careful analysis of an experiment has to be performed in order to interpret the data correctly. In this work, we concentrate on the Higgs contribution to THG response and the influence of nontrivial pairing symmetry. Nevertheless, we calculate the charge-density fluctuations, which dominate the total THG response within our clean-limit analysis. As long as impurity scattering does not introduce any preferred direction, our results about polarizations dependencies should be valid even in the dirty limit.

In this paper, we generalize the analysis of Ref. [49] within the Anderson pseudospin formulation of BCS theory to arbitrary gap symmetry and calculate the induced gap oscillations and the induced current. As already shown in Ref. [61] for a two-band superconductor, the THG intensity contains resonances for each band and for the relative phase oscillation-the Leggett mode. Thus more complex systems can lead to additional features in the spectrum mapping out the underlying structure.

We show, how an arbitrary gap symmetry affects the THG intensity and that composite gap-symmetries can lead to additional resonances. In addition we show how an asymmetric driving scheme may resonate with additional asymmetric Higgs modes leading to multiple resonances in the THG spectrum. Such a driving scheme was inspired by recent experimental findings [52] and tries to model the asymmetry in driving due to small in-plane components of the external field.

This paper is organized as follows. In Sec. II, we introduce the model and explain the time evolution via Bloch equations. In Sec. III, we derive analytic expressions for the driven Higgs oscillations and compare the $s$ - and $d$-wave case. In Sec. IV, we calculate analytic expressions for the THG response as a function of the driving frequency. In Sec. V, we calculate numerically the temperature dependence of the THG response for $s, d$, and $d+s$ wave. The following Sec. VI evaluates the polarization dependence of the THG signal. In Sec. VII, we propose an asymmetric driving scheme and show that an additional resonance peak in the THG signal occurs. Finally we summarize and discuss the results in Sec. VIII. 


\section{HAMILTONIAN AND TIME EVOLUTION}

The starting point of our study is the minimal model for Higgs oscillations, i.e., the mean-field BCS-like Hamiltonian, where we allow a $\boldsymbol{k}$-dependent energy gap

$$
H=\sum_{k \sigma} \epsilon_{k} c_{k \sigma}^{\dagger} c_{k \sigma}-\sum_{k}\left(\Delta_{k} c_{k \uparrow}^{\dagger} c_{-k \downarrow}^{\dagger}+\Delta_{k}^{*} c_{-k \downarrow} c_{k \uparrow}\right) .
$$

Hereby, $\epsilon_{\boldsymbol{k}}=\epsilon_{-\boldsymbol{k}}$ is the energy dispersion measured from the Fermi energy $\epsilon_{\mathrm{F}}, c_{\boldsymbol{k} \sigma}^{\dagger}$ and $c_{\boldsymbol{k} \sigma}$ are the electron creation or annihilation operators and

$$
\Delta_{k}=\sum_{\boldsymbol{k}^{\prime}} V_{k k^{\prime}}\left\langle c_{-\boldsymbol{k}^{\prime} \downarrow} c_{\boldsymbol{k}^{\prime} \uparrow}\right\rangle=\Delta f_{k}
$$

the momentum-dependent energy gap, where we assume a separable pairing interaction $V_{\boldsymbol{k} \boldsymbol{k}^{\prime}}=V f_{\boldsymbol{k}} f_{\boldsymbol{k}^{\prime}}$ with strength $V$ and the symmetry function $f_{k}$ such that

$$
\Delta=V \sum_{k} f_{k}\left\langle c_{-k \downarrow} c_{k \uparrow}\right\rangle
$$

Hereby, we assume that $f_{k}$ is normalized to 1 . With this, $\Delta$ corresponds to the maximum of the absolute value of the gap. We implicitly restrict all summations over $\boldsymbol{k}$ in the range $-\boldsymbol{k}_{c}<\boldsymbol{k}<\boldsymbol{k}_{c}$ with the momentum cutoff $\boldsymbol{k}_{c}$ and number of $\boldsymbol{k}$-points $N$. For the sake of simplicity we will restrict all of our calculations to two dimensions having also the quasi-2d layered cuprates in mind. Coupling to an electric field is incorporated via minimal substitution $\epsilon_{\boldsymbol{k}} \rightarrow \epsilon_{\boldsymbol{k}-e \boldsymbol{A}(t)}$ with the electron charge $e$ and the vector potential

$$
\boldsymbol{A}(t)=\left(\begin{array}{l}
A_{x}(t) \\
A_{y}(t)
\end{array}\right)=A_{0}(t) \hat{e}_{A}=A_{0} \sin (\Omega t)\left(\begin{array}{c}
\cos \theta \\
\sin \theta
\end{array}\right),
$$

where $A_{0}$ is the driving amplitude, $\Omega$ the driving frequency and $\theta$ the polarization angle. The choice of our vector potential is such that its wave vector is perpendicular to the superconducting plane without an in-plane component. This choice matches the experiments done so far for the conventional $s$-wave superconductor $\mathrm{NbN}$ [50,51]. However, small in-plane components could be induced due to nonperfect alignment, tilted laser pulses or higher-order nonlinear couplings leading to an asymmetric driving scheme. This idea and its effects will be addressed in Sec. VII.

For our further analysis we will make use of Anderson pseudospin formalism [62]. We introduce the Nambu-Gorkov spinor $\Psi_{\boldsymbol{k}}^{\dagger}=\left(c_{\boldsymbol{k} \uparrow}^{\dagger} c_{-\boldsymbol{k} \downarrow}\right)$ and define Anderson pseudospin

$$
\sigma_{k}=\frac{1}{2} \Psi_{k}^{\dagger} \tau \Psi_{k}=\frac{1}{2}\left(\begin{array}{c}
c_{-k \downarrow} c_{k \uparrow}+c_{k \uparrow}^{\dagger} c_{-k \downarrow}^{\dagger} \\
i\left(c_{-k \downarrow} c_{k \uparrow}-c_{k \uparrow}^{\dagger} c_{-k \downarrow}^{\dagger}\right) \\
c_{k \uparrow}^{\dagger} c_{k \uparrow}-c_{-k \downarrow} c_{-k \downarrow}^{\dagger}
\end{array}\right)
$$

with $\tau$ the vector of Pauli matrices. The BCS Hamiltonian can then be rewritten in terms of the pseudospin and takes the form of a Hamiltonian for a magnetic spin in an external magnetic field

$$
H=\sum_{k} b_{k} \sigma_{k}
$$

with the definition of the pseudomagnetic field

$$
\boldsymbol{b}_{\boldsymbol{k}}=\left(\begin{array}{c}
-2 \Delta^{\prime} f_{\boldsymbol{k}} \\
2 \Delta^{\prime \prime} f_{\boldsymbol{k}} \\
\epsilon_{\boldsymbol{k}-e \boldsymbol{A}(t)}+\epsilon_{\boldsymbol{k}+e \boldsymbol{A}(t)}
\end{array}\right)
$$

and $\Delta=\Delta^{\prime}+i \Delta^{\prime \prime}$. The gap equation takes the form

$$
\Delta=V \sum_{k} f_{k}\left(\left\langle\sigma_{k}^{x}\right\rangle-i\left\langle\sigma_{k}^{y}\right\rangle\right)
$$

In the equilibrium situation with $A_{0}=0$ at temperature $T$, all the pseudospins are aligned parallel to the pseudomagnetic field. Therefore the equilibrium expectation values of the pseudospins read

$$
\begin{gathered}
\left\langle\sigma_{k}^{x}\right\rangle(0)=\frac{\Delta f_{k}}{2 E_{k}} \tanh \left(\frac{E_{k}}{2 k_{B} T}\right), \\
\left\langle\sigma_{k}^{y}\right\rangle(0)=0, \\
\left\langle\sigma_{k}^{z}\right\rangle(0)=-\frac{\epsilon_{k}}{2 E_{k}} \tanh \left(\frac{E_{k}}{2 k_{B} T}\right), \\
\Delta(0)=\Delta=V \sum_{k} f_{k}\left\langle\sigma_{k}^{x}\right\rangle(0)
\end{gathered}
$$

with the quasiparticle energy

$$
E_{k}=\sqrt{\epsilon_{k}^{2}+\left(\Delta f_{k}\right)^{2}} .
$$

Hereby, we assumed a real equilibrium gap, i.e., $\Delta^{\prime \prime}=0$ and $\Delta=\Delta^{\prime}$. We use the following ansatz to describe the deviations $x_{k}(t), y_{k}(t), z_{k}(t)$, and $\delta \Delta(t)$ from the equilibrium pseudospin expectation values and the gap

$$
\begin{gathered}
\left\langle\sigma_{k}^{x}\right\rangle(t)=\left\langle\sigma_{k}^{x}\right\rangle(0)+x_{k}(t), \\
\left\langle\sigma_{k}^{y}\right\rangle(t)=\left\langle\sigma_{k}^{y}\right\rangle(0)+y_{k}(t), \\
\left\langle\sigma_{k}^{z}\right\rangle(t)=\left\langle\sigma_{k}^{z}\right\rangle(0)+z_{k}(t), \\
\Delta(t)=\Delta+\delta \Delta(t), \\
\delta \Delta(t)=V \sum_{k} f_{k}\left(x_{k}(t)-i y_{k}(t)\right) .
\end{gathered}
$$

The time evolution of the expectation values is governed by Heisenbergs' equation of motion

$$
\partial_{t} \sigma_{k}(t)=i\left[H, \sigma_{k}\right]
$$

which take the form of Bloch equations

$$
\left(\begin{array}{c}
\dot{x}_{k}(t) \\
\dot{y}_{k}(t) \\
\dot{z}_{k}(t)
\end{array}\right)=b_{k}(t) \times\left\langle\sigma_{k}\right\rangle(t) .
$$

Note that we have set $\hbar=1$ here and in the following.

For the further calculation, we expand the $z$ component of the pseudomagnetic field up to second order in $A_{0}$

$$
\epsilon_{\boldsymbol{k}-e A(t)}+\epsilon_{\boldsymbol{k}+e A(t)}=2 \epsilon_{\boldsymbol{k}}+\epsilon_{\boldsymbol{k}}^{A}(t)+\mathcal{O}\left(A_{0}^{4}\right)
$$

with the definition

$$
\epsilon_{\boldsymbol{k}}^{A}(t)=e^{2} \sum_{i j} A_{i}(t) A_{j}(t) \partial_{i j}^{2} \epsilon_{k},
$$


where we used the short notation $\partial_{i j}^{2} \epsilon_{k}=\frac{\partial^{2} \epsilon_{k}}{\partial_{k_{i}} k_{k}}$. Due to the assumption $\epsilon_{\boldsymbol{k}}=\epsilon_{-\boldsymbol{k}}$, the term linear in $A_{0}$ vanishes and only the quadratic coupling remains. Written out explicitly, we find

$$
\epsilon_{k}^{A}(t)=e^{2} A_{0}^{2}(t) D_{\epsilon_{k}}(\theta)
$$

with

$$
\begin{aligned}
D_{\epsilon_{k}}(\theta)= & \cos ^{2} \theta \partial_{x x}^{2} \epsilon_{k}+\sin ^{2} \theta \partial_{y y}^{2} \epsilon_{k} \\
& +2 \sin \theta \cos \theta \partial_{x y}^{2} \epsilon_{k} .
\end{aligned}
$$

The term $D_{\epsilon_{k}}(\theta)$ contains the polarization dependence and the second derivatives of the dispersion. We can see that a nonparabolicity is crucial for the coupling as a parabolic dispersion would lead to a $\boldsymbol{k}$-independent coupling term describing only a time-dependent variation of the Fermi energy. This changes in the dirty limit, where also the paramagnetic coupling term remains leading to a much stronger coupling $[59,60]$. The explicit form of the Bloch equations used in the following reads

$$
\left\langle\dot{\boldsymbol{\sigma}}_{\boldsymbol{k}}\right\rangle(t)=\left(\begin{array}{c}
-2\left(\Delta+\delta \Delta^{\prime}(t)\right) f_{k} \\
2 \delta \Delta^{\prime \prime}(t) f_{k} \\
2 \epsilon_{\boldsymbol{k}}+\epsilon_{\boldsymbol{k}}^{A}(t)
\end{array}\right) \times\left(\begin{array}{c}
\left\langle\sigma_{\boldsymbol{k}}^{x}\right\rangle(0)+x_{k}(t) \\
y_{\boldsymbol{k}}(t) \\
\left\langle\sigma_{\boldsymbol{k}}^{z}\right\rangle(0)+z_{\boldsymbol{k}}(t)
\end{array}\right) .
$$

\section{HIGGS OSCILLATIONS}

To gain a first insight into the driven dynamics and see the differences to the known $s$-wave case, we start by deriving analytic expressions for the gap oscillations. To this end, we assume a small driving amplitude $e A_{0} \ll 1$, such that we can neglect terms in second order of the deviation from equilibrium. For simpler notation, we will show only the results for the zero temperature case $T=0$. A generalization for finite temperature is straight forward by including the factor $\tanh \left(E_{k} /\left(2 k_{B} T\right)\right)$ in the respective expressions and numerical results for this case are shown in the later sections. The linearized equations of motion read

$$
\begin{aligned}
\dot{x}_{k}(t) & =-2 \epsilon_{k} y_{k}(t)-\frac{f_{k}}{E_{k}} \epsilon_{k} \delta \Delta^{\prime \prime}(t), \\
\dot{y}_{k}(t)= & 2 \epsilon_{k} x_{k}(t)+2 f_{k} \Delta z_{k}(t) \\
& +\frac{f_{k}}{E_{k}}\left(\frac{1}{2} \epsilon_{k}^{A}(t) \Delta-\epsilon_{k} \delta \Delta^{\prime}(t)\right), \\
\dot{z}_{k}(t) & =-2 f \Delta y_{k}(t)-\frac{\Delta f_{k}^{2}}{E_{k}} \delta \Delta^{\prime \prime}(t) .
\end{aligned}
$$

We perform a Laplace transform from time $t$ to complex frequencies $s$ according to

$$
a(t) \rightarrow a(s)=\int_{0}^{\infty} e^{-s t} a(t) d t
$$

to obtain algebraic equations

$$
\begin{aligned}
s x_{k}(s) & =-2 \epsilon_{k} y_{k}(s)-\frac{f_{k}}{E_{k}} \epsilon_{k} \delta \Delta^{\prime \prime}(s), \\
s y_{k}(s)= & 2 \epsilon_{k} x_{k}(s)+2 f_{k} \Delta z_{k}(s) \\
& +\frac{f_{k}}{E_{k}}\left(\frac{1}{2} \epsilon_{k}^{A}(s) \Delta-\epsilon_{k} \delta \Delta^{\prime}(s)\right), \\
s z_{k}(s)= & -2 f \Delta y_{k}(s)-\frac{\Delta f_{k}^{2}}{E_{k}} \delta \Delta^{\prime \prime}(s) .
\end{aligned}
$$

Solving for the deviation terms, we find

$$
\begin{gathered}
x_{k}(s)=\frac{\epsilon_{k} f_{k}\left(2 \epsilon_{k} \delta \Delta^{\prime}(s)-\Delta \epsilon_{k}^{A}(s)-s \delta \Delta^{\prime \prime}(s)\right)}{E_{k}\left(4 E_{k}^{2}+s^{2}\right)}, \\
y_{k}(s)=\frac{-f_{k}\left(2 s \epsilon_{k} \delta \Delta^{\prime}(s)-s \Delta \epsilon_{k}^{A}(s)+4 E_{k}^{2} \delta \Delta^{\prime \prime}(s)\right)}{2 E_{k}\left(4 E_{k}^{2}+s^{2}\right)}, \\
z_{k}(s)=\frac{\Delta f_{k}^{2}\left(2 \epsilon_{k} \delta \Delta^{\prime}(s)-\Delta \epsilon_{k}^{A}(s)-s \delta \Delta^{\prime \prime}(s)\right)}{E_{k}\left(4 E_{k}^{2}+s^{2}\right)} .
\end{gathered}
$$

To proceed with a further analytic analysis we use several assumptions and approximations, e.g., isotropy in $x$ and $y$ directions (for details see Appendix A). With these assumptions, we can simplify the interaction term $\epsilon_{k}^{A}(t)$ under a summation with an arbitrary factor $a_{k}$ which becomes polarization independent

$$
\sum_{k} \epsilon_{k}^{A}(t) a_{k}=\sum_{k} e^{2} A_{0}^{2}(t)\left(\alpha_{0}+\alpha_{1} \epsilon_{k}\right) a_{k} .
$$

Hereby, $\alpha_{i}$ are expansion coefficients. To evaluate the momentum sum in the gap equation Eq. (8), we use the approximation that the dispersion depends only on the absolute value of $\boldsymbol{k}$ near $\epsilon_{\mathrm{F}}$, i.e., $\epsilon_{\boldsymbol{k}}=\epsilon(|\boldsymbol{k}|)$, whereas the gap symmetry function only depends on the polar angle $\varphi$, i.e., $f_{k}=f(\varphi)$. Then, we can replace the momentum sum with an integral over the energy $\epsilon$ and an integral over the polar angle $\varphi$

$$
V \sum_{k} \rightarrow \lambda \int_{-\epsilon_{c}}^{\epsilon_{c}} d \epsilon \int_{0}^{2 \pi} d \varphi
$$

with $\lambda=V D\left(\epsilon_{\mathrm{F}}\right)$, where the density of states $D\left(\epsilon_{\mathrm{F}}\right)$ is assumed to be constant near the Fermi energy. In the following, we will replace the integral borders of the $\epsilon$ integral with $\pm \infty$ which is valid for $\epsilon_{c} \gg \Delta$. For further notation simplification, we will drop the energy and angular dependencies of the functions in the integrands.

Next, we insert the expressions for $x_{k}(t)$ and $y_{k}(t)$ into the real and imaginary part of the gap equation

$$
\begin{gathered}
\delta \Delta^{\prime}(s)=\lambda \int d \epsilon \int d \varphi f x(s), \\
\delta \Delta^{\prime \prime}(s)=-\lambda \int d \epsilon \int d \varphi f y(s) .
\end{gathered}
$$

We start by solving the equation for the real part of the gap. The terms $\propto \delta \Delta^{\prime \prime}(s)$ and $\propto \alpha_{0}$ in Eq. (38) vanish as

$$
\int \frac{\epsilon}{E\left(4 E^{2}+s^{2}\right)} d \epsilon=0
$$

due to the asymmetry of the integrand. The resulting expression reads

$$
\begin{aligned}
\delta \Delta^{\prime}(s)= & \frac{1}{2} \alpha_{1} \Delta e^{2} A_{0}^{2}(s) \\
& \times\left(1-\frac{1}{\lambda \int f^{2}\left(4 \Delta^{2} f^{2}+s^{2}\right) F(s, \varphi) d \varphi}\right) .
\end{aligned}
$$

To obtain this result we identified the equilibrium gap equation

$$
1=\lambda \int d \epsilon \int d \varphi \frac{f^{2}}{2 E}
$$


by adding a zero $4 \Delta^{2} f^{2}+s^{2}-4 \Delta^{2} f^{2}-s^{2}$ in the nominator of the respective expression in the integrand. The function $F(s, \varphi)$ is defined by

$$
\begin{aligned}
F(s, \varphi) & =\int \frac{1}{2 E\left(4 E^{2}+s^{2}\right)} d \epsilon \\
& =\frac{1}{s \sqrt{4 \Delta^{2} f^{2}+s^{2}}} \sinh ^{-1}\left(\frac{s}{2 \Delta|f|}\right) .
\end{aligned}
$$

Next, we use

$$
A_{0}^{2}(s)=A_{0}^{2} \frac{2 \Omega^{2}}{s\left(s^{2}+4 \Omega^{2}\right)}
$$

and define

$$
\begin{aligned}
& I_{1}(s)=\frac{\Omega^{2}}{s\left(s^{2}+4 \Omega^{2}\right)}, \\
& I_{2}(s)= \frac{\Omega^{2}}{\left(s^{2}+4 \Omega^{2}\right)} \\
& \times \frac{1}{\lambda \int f^{2} \sqrt{4 \Delta^{2} f^{2}+s^{2}} \sinh ^{-1}\left(\frac{s}{2 \Delta|f|}\right) d \varphi}
\end{aligned}
$$

to write $\delta \Delta^{\prime}(s)=\alpha_{1} \Delta e^{2} A_{0}^{2}\left(I_{1}(s)+I_{2}(s)\right)$. The solution in the time domain is obtained by performing the inverse Laplace transform. The calculation can be found in the Appendix B. The solution $\delta \Delta^{\prime}(t)$ consists of three contributions

$$
\delta \Delta^{\prime}(t)=\alpha_{1} \Delta e^{2} A_{0}^{2}\left(\delta \Delta_{D}(t)+\delta \Delta_{H}(t)+\delta \Delta_{R}(t)\right),
$$

where

$$
\begin{aligned}
& \delta \Delta_{D}(t)=\frac{1-\cos (2 \Omega t)}{4}, \\
& \delta \Delta_{H}(t)= \frac{1}{\pi} \int_{-2 \Delta}^{2 \Delta} d r \frac{\Omega^{2}}{4 \Omega^{2}-r^{2}} \\
& \times \frac{\sin (r t)}{\lambda \int d \varphi f^{2} \sqrt{4 \Delta^{2} f^{2}-r^{2}} \sin ^{-1}\left(\frac{r}{2 \Delta|f|}\right)} \\
&-\frac{1}{4 \lambda \int f^{2} d \varphi}, \\
& \delta \Delta_{R}(t)= \frac{\Omega \cos (2 \Omega t)}{4 \lambda \int d \varphi f^{2} \sqrt{\Delta^{2} f^{2}-\Omega^{2}} \sin ^{-1}\left(\frac{\Omega}{\Delta|f|}\right)} .
\end{aligned}
$$

The term $\delta \Delta_{D}(t)$ is a forced oscillation of the gap with twice the driving frequency $\Omega$ due to the nonlinear $A(t)^{2}$ driving. The term $\delta \Delta_{H}(t)$ is the intrinsic Higgs oscillation with a frequency of $\sim 2 \Delta$ induced by an effective interaction quench resulting from the periodic driving [49]. The amplitude of the Higgs oscillation depends on the driving frequency $\Omega$. Without solving the integral explicitely, we can see that there will be a resonance when $\Omega=\Delta$ due to the prefactor $\Omega^{2} /\left(4 \Omega^{2}-r^{2}\right)$. We can understand this as a coincidence of the two oscillations $2 \Omega$ and $2 \Delta$, when $\Omega$ is tuned to $\Delta$. This resonance can be found also in the third term, which is again a forced oscillation of $2 \Omega$ where the amplitude is also frequency dependent. We rewrite it as

$$
\delta \Delta_{R}(t)=\frac{1}{4 \lambda} A(\Omega) e^{-i \zeta(\Omega)} \cos (2 \Omega t)
$$

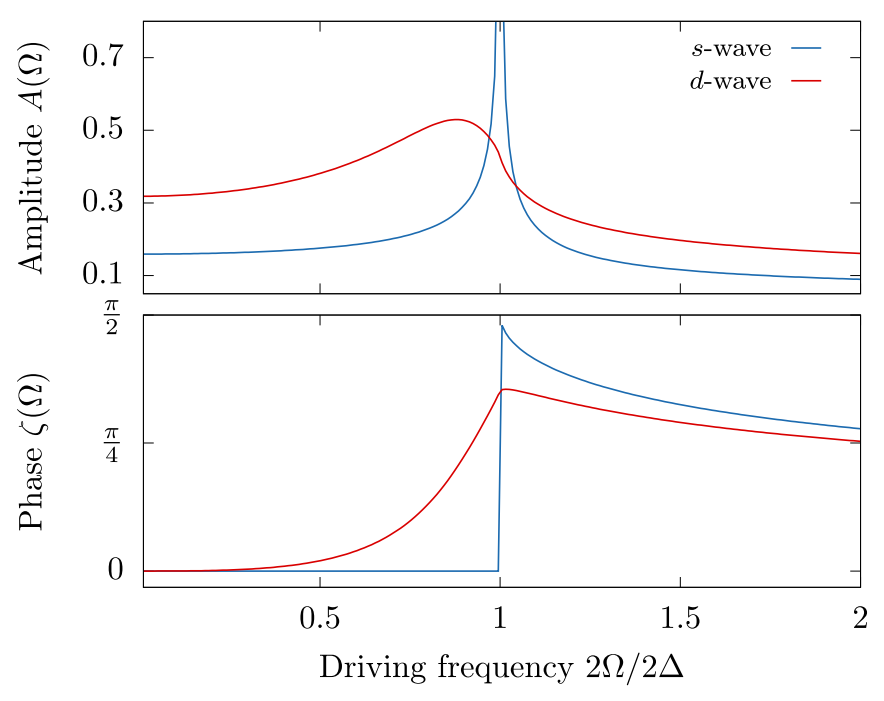

FIG. 2. Amplitude $A$ and phase $\zeta$ of the driven gap oscillation (52). In the case of $s$ wave, a sharp resonance at $2 \Omega=2 \Delta$ occurs, whereas for $d$ wave, a broad peak slightly below $2 \Delta$ is apparent. In addition, the phase of $s$ wave shows a sharp jump from 0 for $2 \Omega<$ $2 \Delta$ to $\pi / 2$ at $2 \Omega=2 \Delta$ and a drifting for $2 \Omega>2 \Delta$. For $d$ wave, there is a broad phase change from 0 for $\Omega=0$ up to a value slightly below $\pi / 2$ for $2 \Omega=2 \Delta$. At this point there is a sharp kink and a drifting for $2 \Omega>2 \Delta$ similar to the $s$-wave case.

where $A(\Omega)$ represents the oscillation amplitude and $\zeta(\Omega)$ the phase relative to the driving.

Let us first recapitulate the known result for the $s$-wave case [49], where $f(\varphi)=1$ and $\lambda \rightarrow \lambda /(2 \pi)$. In this case, there are two isolated branch points at $s= \pm 2 i \Delta$ which lead to an exact resonance in the amplitude if $2 \Omega=2 \Delta$ (see Appendix B for details), i.e., the driving frequency resonates with the intrinsic Higgs oscillation at $2 \Delta$

$$
A(\Omega)=\frac{\Omega}{\sqrt{\Delta^{2}-\Omega^{2}}} \frac{1}{\sin ^{-1}\left(\frac{\Omega}{\Delta}\right)} .
$$

The amplitude $A(\Omega)$ and phase $\zeta(\Omega)$ of the resonance term is plotted in Fig. 2. One can observe a sharp resonance and also a sharp $\pi / 2$ phase jump at the resonance condition.

Next, let us consider the $d$-wave case where $f(\varphi)=$ $\cos (2 \varphi)$. Here, we do no longer have an exact resonance condition but there is still a maximum of the amplitude given by the minimum of the integral in the denominator of (51). This leads to a broad peak in the amplitude accompanied by a smooth phase change of $\zeta(0)-\zeta(2 \Delta)<\pi / 2$. The peak in the amplitude is at an energy slightly below $2 \Omega=2 \Delta$, whereas are sharp kink in the phase at $2 \Omega=2 \Delta$ is found.

For nontrivial gap symmetry, the gap oscillation spectrum does not have to be peaked at exactly $2 \Delta$. It depends on several factors including the density of states or the exact shape of the gap symmetry function. In particular, here, the position of the peak is determined by the interplay between the terms $f^{2}$ and $\sqrt{\Delta^{2} f^{2}-\Omega^{2}}$ under the integral over $\varphi$ in (51). For values $\Omega \approx \Delta$, the terms with the highest weights, i.e., $f \approx 1$, vanish which decreases the value of the integral and creates its minimum. Therefore, in the case of $d$ wave, the maximum of the amplitude can be still found roughly at 
$2 \Omega \approx 2 \Delta$. Depending on the exact shape of $f$, it can be shifted to lower energies. For values $2 \Omega>2 \Delta$, the square root is always imaginary for each value of $\varphi$, which leads to the sharp edge in the phase at $2 \Omega=2 \Delta$.

After studying the real part, we will finally also calculate the imaginary part of the gap with Eq. (39). Similar to the real part, the terms $\propto \delta \Delta^{\prime}(s)$ and $\propto \alpha_{1}$ vanish and we are left with

$$
\begin{aligned}
\delta \Delta^{\prime \prime}(s)= & -\alpha_{0} \Delta e^{2} A_{0}^{2}(s) s \lambda \int d \varphi f^{2} F(s, \varphi) \\
& +\delta \Delta^{\prime \prime}(s)-\delta \Delta^{\prime \prime}(s) s^{2} \lambda \int d \varphi f^{2} F(s, \varphi) .
\end{aligned}
$$

Solving for $\delta \Delta^{\prime \prime}(s)$ yields

$$
\delta \Delta^{\prime \prime}(s)=-\alpha_{0} \Delta e^{2} A_{0}^{2} \frac{2 \Omega}{s^{2}\left(s^{2}+4 \Omega^{2}\right)}
$$

and the solution in the time domain is given by

$$
\begin{aligned}
\delta \Delta^{\prime \prime}(t) & =-\alpha_{0} \Delta e^{2} A_{0}^{2}\left(\frac{t}{2}-\frac{\sin (2 \Omega t)}{4 \Omega}\right) \\
& =-\alpha_{0} \Delta e^{2} \int_{0}^{t} A_{0}\left(t^{\prime}\right)^{2} d t^{\prime} .
\end{aligned}
$$

We can see that the imaginary part is independent of the gap symmetry. It follows the oscillation of the driving frequency with a frequency independent amplitude. In addition it contains a drift linear in time. Like in the $s$-wave case, for small driving amplitudes, the imaginary part only contributes to the phase $\varphi_{\Delta}$ of the gap $\Delta(t)=|\Delta(t)| e^{i \varphi_{\Delta}(t)}$

$$
\varphi_{\Delta}(t)=\arctan \left(\frac{\delta \Delta^{\prime \prime}(t)}{\Delta+\delta \Delta^{\prime}(t)}\right) \approx \frac{\delta \Delta^{\prime \prime}(t)}{\Delta} .
$$

The absolute value of the gap is independent of the imaginary part and is solely determined by the real part

$$
|\Delta(t)|=\sqrt{\left(\Delta+\delta \Delta^{\prime}(t)\right)^{2}+\delta \Delta^{\prime \prime}(t)^{2}} \approx \Delta+\delta \Delta^{\prime}(t) .
$$

\section{THIRD HARMONIC GENERATION}

The resonance of the forced gap oscillation with the Higgs mode can be found experimentally in the transmitted light field. The nonlinear coupling of the vector potential to the condensate leads to higher-harmonic generation where the lowest nonvanishing order is third-harmonic generation. Its intensity $I^{\mathrm{THG}}$, which is proportional to the amplitude squared of the induced current $j^{(3)}(3 \Omega)$

$$
I^{\mathrm{THG}} \propto\left|j^{(3)}(3 \Omega)\right|^{2}
$$

shows the same resonance as the gap oscillation and will be calculated in the following. However, not only the driven gap oscillations contribute to THG but also charge density fluctuations, i.e., single-particle excitations resonating at the pair-breaking energy $2 \Delta$. In the clean limit, depending on material parameters, these can be significantly larger in an experiment and overlay the contribution from the gap oscillation. Therefore this contribution is considered and calculated in this section as well and compared to the contribution from the Higgs oscillation.
Driving the superconductor periodically will induce an electric current

$$
\boldsymbol{j}(t)=e \sum_{\boldsymbol{k}} \boldsymbol{v}_{\boldsymbol{k}-e \boldsymbol{A}(t)}\left\langle n_{\boldsymbol{k}}\right\rangle(t)
$$

with the group velocity $\boldsymbol{v}_{k}=\nabla \epsilon_{k}$ and the charge density

$$
\left\langle n_{\boldsymbol{k}}\right\rangle(t)=\left\langle c_{\boldsymbol{k} \uparrow}^{\dagger} c_{\boldsymbol{k} \uparrow}+c_{\boldsymbol{k} \downarrow}^{\dagger} c_{\boldsymbol{k} \downarrow}\right\rangle(t) .
$$

To calculate the lowest order response, we expand the velocity in $A_{0}$

$$
v_{k-e A(t)}=\boldsymbol{v}_{\boldsymbol{k}}-e \sum_{j} A_{j}(t) \partial_{j} \boldsymbol{v}_{\boldsymbol{k}}+\mathcal{O}\left(A_{0}^{2}\right)
$$

The charge density can be expressed with the $z$-component of the pseudospin and we obtain for the current

$$
\boldsymbol{j}(t)=\boldsymbol{j}^{(0)}(t)+\boldsymbol{j}^{(1)}(t)+\boldsymbol{j}^{(3)}(t),
$$

where

$$
\begin{gathered}
\boldsymbol{j}^{(0)}(t)=e \sum_{\boldsymbol{k}} \boldsymbol{v}_{\boldsymbol{k}}\left\langle n_{\boldsymbol{k}}\right\rangle(t) \\
\boldsymbol{j}^{(1)}(t)=-2 e^{2} \sum_{\boldsymbol{k}} \sum_{j} A_{j}(t) \partial_{j} \boldsymbol{v}_{\boldsymbol{k}}\left(\left\langle\sigma_{\boldsymbol{k}}^{z}\right\rangle(0)+\frac{1}{2}\right) \\
\boldsymbol{j}^{(3)}(t)=-2 e^{2} \sum_{\boldsymbol{k}} \sum_{j} A_{j}(t) \partial_{j} \boldsymbol{v}_{\boldsymbol{k}} z_{\boldsymbol{k}}(t)
\end{gathered}
$$

The first term $j^{(0)}(t)$ vanishes due to parity, the second term $\boldsymbol{j}^{(1)}(t)$ represents the induced current oscillating with the driving frequency $\Omega$. The third term $j^{(3)}(t)$ is the lowest order of higher-order generation, which oscillates with $3 \Omega$ due to the proportionality $\propto A_{j}(t) z_{k}(t)$ as we have seen in the previous section that $z_{k}(t)$ oscillates with $2 \Omega$. The induced current for an arbitrary angle relative to the polarization of the vector potential can be decomposed into a parallel and perpendicular component which we will calculate separately. We insert the expression for the vector potential and expand the summation of the components

$$
\begin{aligned}
j_{\| \perp}^{(3)}(t) & =j^{(3)}(t) \cdot \hat{e}_{A}^{\| \perp} \\
& =-2 e^{2} A_{0}(t) \sum_{k} D_{\epsilon_{k}}^{\| \perp}(\theta) z_{k}(t)
\end{aligned}
$$

with $\hat{e}_{A}^{\|}=\hat{e}_{A}, \quad \hat{e}_{A}^{\perp}=(\sin \theta,-\cos \theta)^{\top}, \quad D_{\epsilon_{k}}^{\|}=D_{\epsilon_{k}} \quad$ from Eq. (24) and

$$
\begin{aligned}
D_{\epsilon_{k}}^{\perp}= & \sin \theta \cos \theta\left(\partial_{x x}^{2} \epsilon_{k}-\partial_{y y}^{2} \epsilon_{k}\right) \\
& +\partial_{x y}^{2} \epsilon_{k}\left(\sin ^{2} \theta-\cos ^{2} \theta\right) .
\end{aligned}
$$

To obtain an expression for the spectrum, we perform a Fourier transform and make use of the solution $z_{k}(s=i \omega)$ from the linearized Bloch equations (35). There are three different contributions originating from the terms $\propto \delta \Delta^{\prime}(s)$, $\propto \delta \Delta^{\prime \prime}(s)$, and $\propto \epsilon_{k}^{A}(s)$ such that we can write

$$
j_{\| \perp}^{(3)}(3 \Omega)=j_{\| \perp}^{(3) \mathrm{H}}(3 \Omega)+j_{\| \perp}^{(3) \mathrm{P}}(3 \Omega)+j_{\| \perp}^{(3) \mathrm{CDF}}(3 \Omega),
$$


where

$$
\begin{gathered}
j_{\| \perp}^{(3) \mathrm{H}}(3 \Omega) \propto \Delta A_{0} \delta \Delta^{\prime}(2 i \Omega), \\
j_{\| \perp}^{(3) \mathrm{P}}(3 \Omega) \propto \Delta A_{0} \delta \Delta^{\prime \prime}(2 i \Omega), \\
j_{\| \perp}^{(3) \mathrm{CDF}}(3 \Omega) \propto \Delta^{2} A_{0} A_{0}^{2}(2 i \Omega) .
\end{gathered}
$$

Details of the calculation and exact expression can be found in Appendix C. The first term (72) is the Higgs contribution following from the amplitude oscillation of the order parameter. The second term (73) follows from the oscillation of the imaginary part of the gap, i.e., oscillations of the phase. The third term (74) depends on the coupling of the vector potential to the band dispersion and describes charge density fluctuations as the expression has the form of a density-density correlation function [53].

Using the same assumptions from the previous section and rewriting occurring sums as integrals, one obtains

$$
\begin{aligned}
j_{\|}^{(3) \mathrm{H}}(3 \Omega) \propto & \alpha_{1}^{2} \Delta^{2} e^{2} A_{0}^{2}(2 i \Omega) \\
& \times\left(1-\frac{H(2 i \Omega)}{2}-\frac{1}{2 H(2 i \Omega)}\right), \\
j_{\|}^{(3) \mathrm{P}}(3 \Omega) \propto \alpha_{0}^{2} \Delta^{2} e^{2} A_{0}^{2}(2 i \Omega) 2 G(2 i \Omega), & \\
j_{\perp}^{(3) \mathrm{CDF}}(3 \Omega) \propto & -\Delta^{2} e^{2} A_{0}^{2}(2 i \Omega) \frac{1}{4} \sin 4 \theta \\
& \times\left[4 \alpha_{0}^{2} G(2 i \Omega)+\alpha_{1}^{2}(1-H(2 i \Omega))\right], \\
j_{\|}^{(3) \mathrm{CDF}}(3 \Omega) \propto & -\Delta^{2} e^{2} A_{0}^{2}(2 i \Omega)\left(1-\frac{1}{2} \sin ^{2} 2 \theta\right) \\
\times & {\left[4 \alpha_{0}^{2} G(2 i \Omega)+\alpha_{1}^{2}(1-H(2 i \Omega))\right] . }
\end{aligned}
$$

For these expressions, we defined the integrals

$$
\begin{gathered}
H(2 i \Omega)=\lambda \int f^{2}\left(4 \Delta^{2} f^{2}-4 \Omega^{2}\right) F(2 i \Omega, \varphi) d \varphi, \\
G(2 i \Omega)=\lambda \int f^{2} F(2 i \Omega, \varphi) d \varphi .
\end{gathered}
$$

Each of the three terms for the current contribute to the THG intensity

$$
I_{\| \perp}^{\mathrm{THG}} \propto\left|j_{\| \perp}^{(3)}(3 \Omega)\right|^{2} .
$$

Within the chosen assumptions, we get an important result, namely that the Higgs and phase contributions are polarization independent and their contributions perpendicular to the polarization vector vanish. In case of the CDF, both parallel and perpendicular contributions exist and are polarization dependent with a characteristic dependency independent of the gap symmetry and dispersion. Both expressions $G(2 i \Omega)$ occurring in the phase and CDF terms as well as $1 / H(2 i \Omega)$ in the Higgs term diverge for $2 \Omega=2 \Delta$ in the $s$-wave case or show a maximum in the $d$-wave case. Therefore the resonance of the amplitude oscillation with the Higgs mode is not the only cause of the peak found in the THG. In particular, the individual strengths are determined by the parameters $\lambda$ and $\alpha_{0}$.

For $\alpha_{0}=0$, e.g., the half-filling case in the tight-binding model, the resonance due to the $G$ term vanishes, which

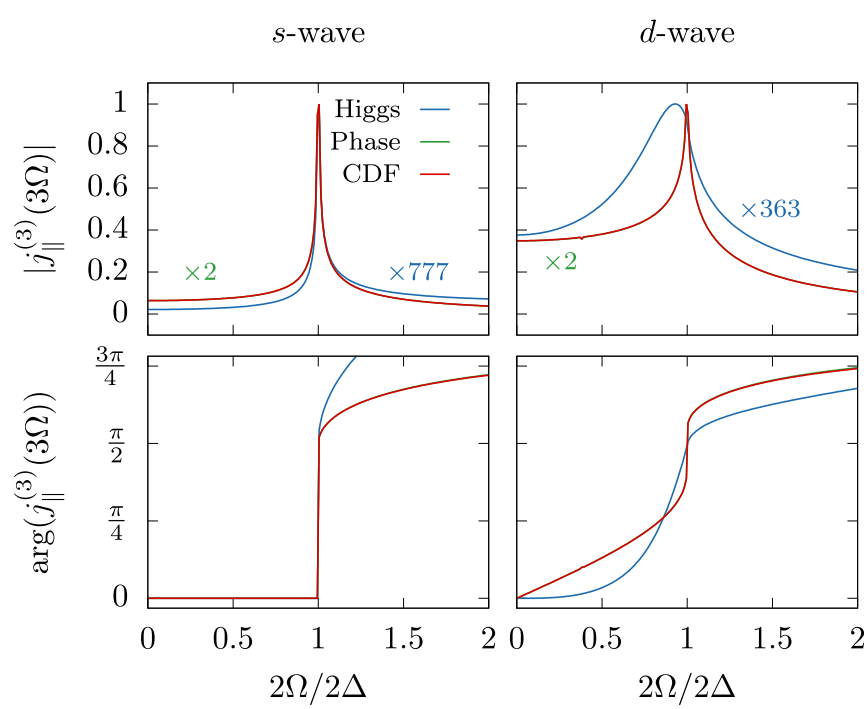

FIG. 3. Individual contributions of the current $j_{\|}^{(3)}(3 \Omega)$ for $s$ and $d$ wave originating from Higgs, CDF and phase oscillations using Eqs. (75), (76), and (78). The phase and CDF contribution lie approximately on top of each other. The parameters are $\Delta=20 \mathrm{meV}$, $\theta=0, \lambda=0.1$ and $\alpha_{0}=-\epsilon_{\mathrm{F}} / 2, \alpha_{1}=-0.5$ with $\epsilon_{\mathrm{F}}=-400 \mathrm{meV}$ assuming the tight-binding dispersion (A3).

removes the phase contribution completely and strongly suppresses the CDF term. For a polarization value of $\theta=\pi / 4$, the CDF contribution perpendicular vanishes and the diverging part due to $G$ in the parallel contribution exactly cancels with the phase contribution. The polarization dependence will be discussed in Sec. VI in more detail. For large $\lambda$, i.e., large interaction strength, the CDF contribution is enhanced over the Higgs contribution. It can be understood in this way that the resonance term for the Higgs contribution scales with $\frac{1}{\lambda}$ due to the $\frac{1}{H}$ term, whereas the resonance term for the CDF contribution scales with $\lambda$ due to the $G$ term. Therefore one can roughly estimate that $j_{\|}^{(3) H}(3 \Omega) \propto 1 / \lambda^{2} j_{\|}^{(3) \mathrm{CDF}}(3 \Omega)$.

In Fig. 3, a comparison of the individual contributions for the $s$ - and $d$-wave cases is shown by evaluating the expressions (75), (76), and (78) for typical parameters. We can see that the CDF term exceeds the Higgs term by more than two orders of magnitude. Within the chosen approximations and parameters, the phase term is roughly $1 / 2$ of the CDF term. Both terms show a sharp peak at $2 \Omega=2 \Delta$ for the $s$ - and $d$-wave cases. This results from the resonance in the $G(2 i \Omega)$ at the pair-breaking energy $2 \Delta$ even for the $d$-wave case due to the much higher weight at the antinodes relative to the nodes. The shape of the Higgs term, originating from the resonance in the amplitude oscillation $1 / H(2 i \Omega)$, follows the shape shown in Fig. 2, i.e., a sharp peak for $s$ wave and a broad peak for $d$ wave. Again for $d$ wave, there is also a lot of weight in the range $2 \Omega<2 \Delta$ as for any $\Omega$ there is always a $\Delta_{k}$, where $2 \Omega=2 \Delta_{k}$ leading to an enhancement of the amplitude. The phase change in the $s$-wave case is very sharp for all contributions, while for the $d$-wave case there are small differences. The phase change in the Higgs term is similar to the phase change of the amplitude oscillation, while the phase change of the CDF and phase term is smooth in the beginning but contains a steep step around $2 \Omega=2 \Delta$. 
Despite the fact that the CDF contribution may exceed the Higgs contribution in our simple analysis, it is still useful for the understanding of the physical mechanisms. The actual weighting of the terms in an experiment depends strongly on the material by further effects not considered in our analysis, like retardation effects in materials with phonon-mediated interaction [54] or the paramagnetic coupling for superconductors in the dirty limit [56-60].

After gaining a first understanding of the terms contributing to the THG intensity under the chosen assumptions for $\epsilon_{k}$ and $f_{k}$, we will drop these in the following sections and solve the summations numerically without approximation and include the temperature dependence. This allows for arbitrary dispersion and gap symmetries to be considered which can introduce new features like additional resonances and polarization dependencies.

\section{TEMPERATURE DEPENDENCE}

In all current experiments so far, the driving frequency cannot be tuned continuously like done in the theoretical analysis from the previous section. In order to find the resonance, the driving frequency is fixed and the temperature is varied until $2 \Omega=2 \Delta(T)$ is fulfilled [50]. For the following, we solve the sums (C3)-(C9) numerically without further approximations. The interaction strength $V$ is calculated for a chosen initial energy gap at $T=0$. Then, the temperature dependence of the energy gap $\Delta(T)$ is determined self-consistently for each temperature. To handle the divergences in the summations and keep the required momentum grid resolution in a reasonable range, we introduced a residual broadening of $2 i \Omega \rightarrow$ $2 i \Omega+0.01 \Delta$. This slightly broadens the resonance peaks and washes out the sharp phase jumps but does not change the overall result. We discretize the momentum space around the Fermi energy with an energy cutoff of $E_{c}=2 \Delta$ with $N_{k}=2000$ points in $k$ and $N_{\varphi}=2000$ points in the angular direction. For the following calculation, we use a $d$-wave gap function $\Delta(\varphi)=\Delta \cos (2 \varphi)$ with $\Delta=20 \mathrm{meV}$ and the tight-binding dispersion (A3) with $t=200 \mathrm{meV}$ and $\epsilon_{\mathrm{F}}=$ $-400 \mathrm{meV}$.

In Fig. 4, the temperature dependence of the THG intensity and phase for $d$ wave in comparison with $s$ wave is shown for different driving frequencies. For driving frequencies $\Omega>$ $\Delta(T=0)$ no resonance occurs and the THG intensity follows roughly the temperature dependence of the energy gap to the power of four, i.e., $\sim \Delta(T)^{4}$. As soon as $\Omega<\Delta(T=0)$, there is a temperature, where $\Omega=\Delta(T)$ and a resonance occurs. There are two main differences in the intensities of $s$ and $d$ wave for the Higgs contribution (dotted lines). First, for the same driving frequency, the resonance peaks are broader for $d$ wave as there is no single resonance point like in the $s$ wave case. Second, due to the continuous variation of the gap from 0 to $\Delta$ for $d$ wave, there is always some resonant gap for temperatures $T<T_{\mathrm{R}}$, where $T_{\mathrm{R}}$ is the temperature for which $\Omega=\Delta\left(T_{\mathrm{R}}\right)$. This leads to a larger THG intensity background in that temperature range. One can also see that for the chosen parameters the CDF contribution dominates as there is still a sharp resonance peak in the total $d$-wave intensity.

Even though the calculation for a pure $s$-wave order parameter compared to a pure $d$-wave order parameter shows a

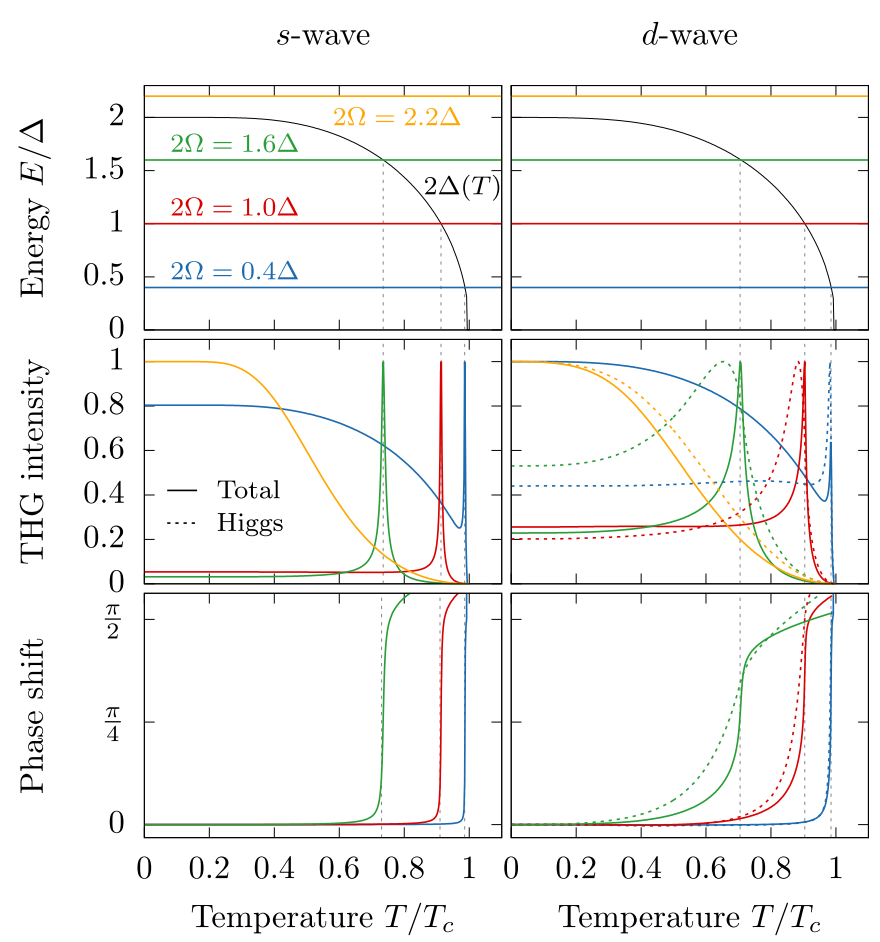

FIG. 4. Temperature dependence of THG intensity for $s$ - and $d$-wave symmetry for four different driving frequencies. The upper row shows the driving frequencies in relation to the energy gap. The middle row shows the THG intensity and the bottom row the phase. The polarization is $\theta=0$. The solid lines are the full THG intensity, the dotted lines only the Higgs contribution. All intensities are normalized to their individual maximum.

clear difference in the peak to background ratio for the Higgs contribution, a comparison of intensities in an experiments is difficult. On the one hand, there is nothing to compare to for a given material. On the other hand, peak intensities can vary due to additional damping effects, which makes a clear distinguishing very unreliable.

The phase analysis confirms the analytic result in Fig. 3. For $s$ wave, a sharp phase jump of $\pi / 2$ occurs at the resonance, whereas for $d$ wave the phase jump is much broader.

As an example for what new features a more exotic gap symmetry may introduce, we will study a case with $d+s$ symmetry, i.e., $d$ wave with an admixture of $s$ wave as it was observed for overdoped YBCO [63-66]. Following Ref. [65], we choose the gap

$$
\Delta f(\varphi)=\Delta_{d} \cos (2 \varphi)+\Delta_{s}
$$

with $\Delta_{d}=0.9 \Delta$ and $\Delta_{s}=0.1 \Delta$.

The temperature dependence of the THG intensity is shown in Fig. 5. As the absolute value of the $d+s$ gap contains two local gap maxima, i.e., $\Delta_{1}^{\max }=\Delta_{d}+\Delta_{s}=\Delta$ and $\Delta_{2}^{\max }=$ $\Delta_{d}-\Delta_{s}=0.8 \Delta$, we show the temperature dependence of these two curves. We can see that if $\Omega<\Delta_{2}^{\max }$, two resonances occur when the driving frequency matches these two maxima. This can be seen both in the THG intensity as two peaks as well as in the phase, where a broad phase transition occurs over the range of the two resonance temperatures with sharp kinks at the resonance points. 


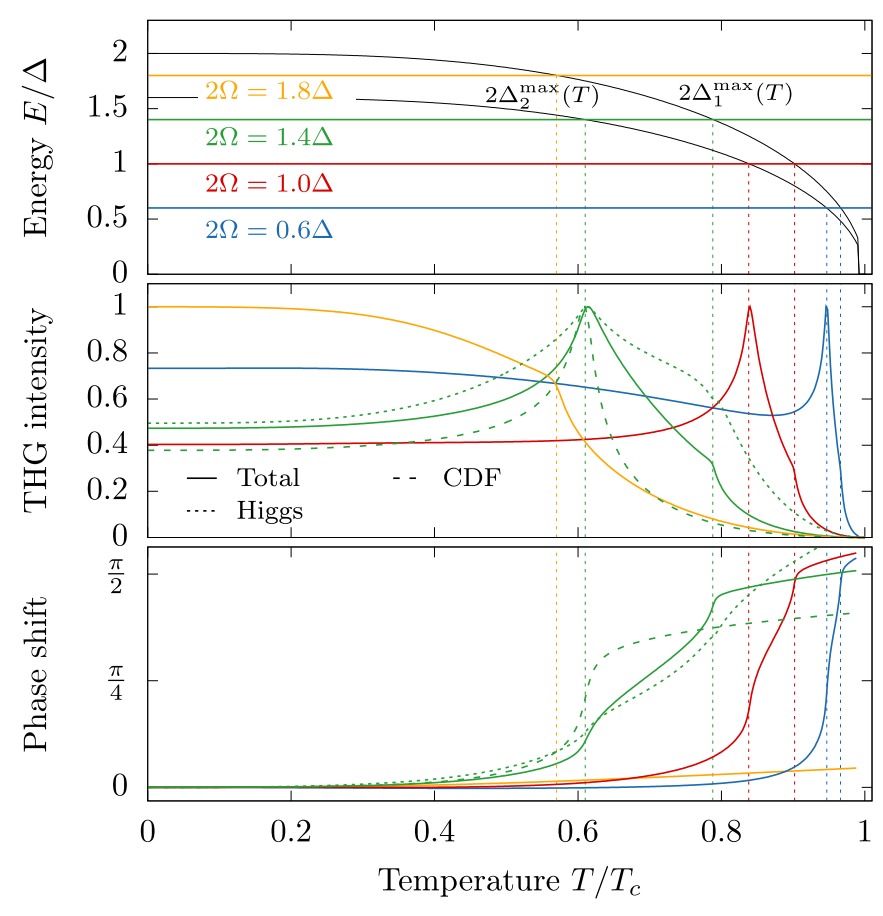

FIG. 5. Temperature dependence of THG intensity for $d+s$ symmetry. The upper row shows the driving frequency in relation to the temperature dependent maxima of the energy gap. The second row shows the total THG intensity with solid lines and exemplary for one driving frequency the respective CDF (dotted) and Higgs (dashed) contribution. The intensity values are individually normalized to their maximum. The third row shows the phase change.

It is interesting to note that the two-peak structure is an effect originating alone from the Higgs contribution despite its smaller value. While the CDF contribution only shows a single peak, it is the Higgs contribution which shows the two-peak structure. We can understand this as two Higgs modes at energies $\Delta_{1}^{\max }$ and $\Delta_{2}^{\max }$ for each local gap maxima which resonate with the driving frequency. This shows that even if the CDF contribution dominates, the Higgs contribution may still contribute to specific features visible in the spectrum. If the polarization is tuned to $\theta=\pi / 4$, the CDF contribution contains a two peak structure as well as the $G$ term in Eq. (78) is suppressed due to the equivalent term with opposite sign in Eq. (76) and the smaller $H$ term with the two-peak structure becomes visible. We can conclude this section by stating that composite gap symmetries can show additional resonances if there are multiple local gap maxima with different amplitudes.

\section{POLARIZATION DEPENDENCE}

As stated above, one possibility in an experiment to gain more insight about the relative weight of Higgs and CDF contributions is the polarization dependence. As one can see in Eq. (78), the CDF contribution has a very characteristic polarization dependence, whereas the Higgs and phase terms do not dependent on the polarization independent on the gap symmetry. If there is no polarization dependence in an experiment it can be a hint that the Higgs contribution is stronger than the CDF part. This was observed in Ref. [51] where it

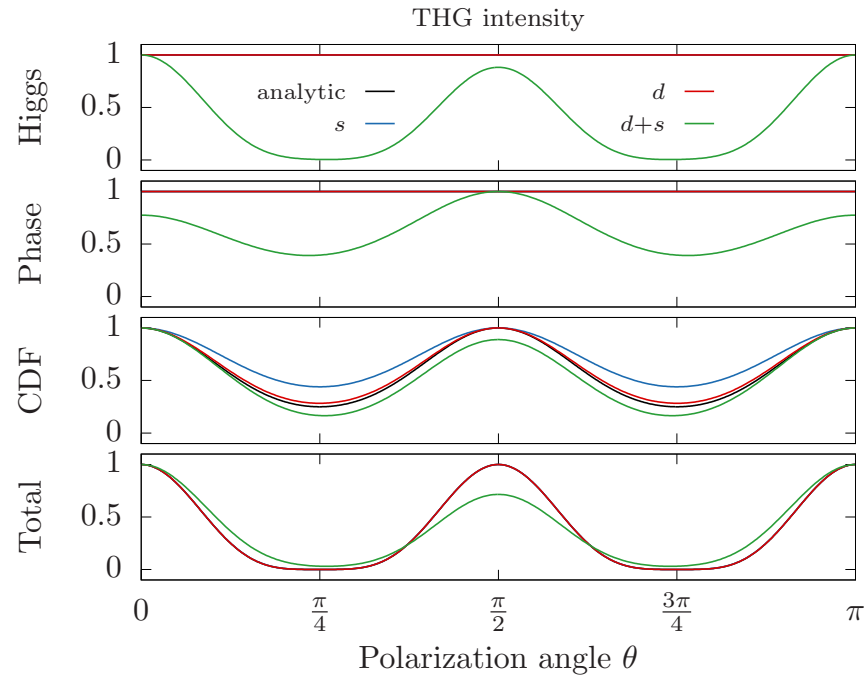

FIG. 6. Polarization dependence of the Higgs contribution (first row), phase contribution (second row), CDF contribution (third row) and total THG intensity (fourth row). The analytic formulas from Eqs. (75)-(78), derived using the assumption of equivalence of $x$ and $y$ directions in $f_{k}^{2}$ and $\epsilon_{k}$, are compared with the numerically calculated results for $s$ and $d$ wave using dispersion Eq. (A3) and $d+$ $s$ wave using dispersion (83) without approximation. All expressions are normalized to their maximum value.

was concluded that in $\mathrm{NbN}$ the Higgs contribution dominates the THG intensity. If we look at the expressions (78) and (76) for the CDF and phase contribution of the current, we can see that for $\theta=\pi / 4$ the $G(2 i \Omega)$ term in the CDF expression cancels exactly with the $G(2 i \Omega)$ term in the phase expression, which means that for this particular polarization angle only the Higgs contribution remains.

However, the derived formulas for the current are only valid under the chosen special assumptions $f_{k}^{2}$ and $\epsilon_{k}$ are symmetric under the exchange of $k_{x} \leftrightarrow k_{y}$, as it is the case for $s$ - or $d$-wave symmetry and a tight-binding dispersion. For the $d+s$ case, this is no longer valid. In Fig. 6 , the polarization dependence for $s, d$, and $d+s$ symmetry calculated numerically is shown in comparison with the analytic result. To show the influence of the dispersion, we use a slightly distorted square lattice dispersion in the $d+s$-wave case

$$
\begin{aligned}
\epsilon_{k}= & -2 t\left(\left(1+\delta_{0}\right) \cos k_{x}+\left(1-\delta_{0}\right) \cos k_{y}\right) \\
& -4 t^{\prime} \cos k_{x} \cos k_{y}-\epsilon_{\mathrm{F}}
\end{aligned}
$$

with $\delta_{0}=-0.03$ and $t=200 \mathrm{meV}, t^{\prime}=-80 \mathrm{meV}$, and $\epsilon_{\mathrm{F}}=$ $-240 \mathrm{meV}$ [65]. One can see that for $s$ and $d$ wave the result closely follows the approximation, i.e., the Higgs contribution is polarization independent and the CDF contribution has a $\propto 1-\frac{1}{2} \sin ^{2} 2 \theta$ dependency with a small offset resulting from the chosen approximations. This changes for $d+s$, where the Higgs and phase terms also becomes polarization dependent and the polarization dependence of the total THG intensity deviates from the analytic formula. Choosing a different dispersion can lead to another polarization dependence. We can see that the polarization dependence alone is not enough to unambiguously distinguish between Higgs and CDF contributions if the band dispersion is not exactly known. This was 
also concluded in Ref. [55], where it was shown that the Higgs contribution can get polarization dependent as well while on the other hand the polarization dependence of the CDF contribution can be suppressed. Additional deviations from the derived formulas for the polarization dependence are possible, e.g., due to interplay with the pseudogap phase or multiband models. This is however beyond the scope of this paper.

\section{ASYMMETRIC DRIVING}

In an experiment, the coupling of light to the superconducting condensate may contain more subtleties and consists not only of a symmetric diamagnetic $A^{2}$ term. For examples, small in-plane components of the wave vector induced by non perfect perpendicular alignment of laser and crystal or on purpose tilted lasers as well as higher order couplings to other finite-momentum modes may induce an asymmetry while driving. Such a momentum dependent driving can lead to asymmetric oscillations of the condensate with respect to the origin. One finds [42] that an asymmetric oscillation of the condensate can show up as a second frequency in the gap oscillation below the well-known $2 \Delta$ Higgs mode. This asymmetric Higgs mode depends on the gap symmetry and the respective asymmetric deviation.

We propose a phenomenological asymmetric driving scheme to describe such effects in an experiment. Due to a momentum dependent driving, the gap symmetry gets altered by an additional symmetry component $f_{k}^{A}$. We add such a term to the pseudomagnetic field altering the gap symmetry with the same time dependence as the usual driving term

$$
\boldsymbol{b}_{k}=\left(\begin{array}{c}
-2 \Delta^{\prime}\left(f_{k}+\delta_{A} \sin ^{2}(\Omega t) f_{\boldsymbol{k}}^{A}\right) \\
2 \Delta^{\prime \prime}\left(f_{\boldsymbol{k}}+\delta_{A} \sin ^{2}(\Omega t) f_{\boldsymbol{k}}^{A}\right) \\
2 \epsilon_{\boldsymbol{k}}+\epsilon_{\boldsymbol{k}}^{A}(t)
\end{array}\right),
$$

where $\delta_{A}$ determines the strength of the asymmetric driving. There is some similarity to [7], where, however static and $a b$ initio, a composite pairing interaction leads to multiple Higgs modes in the different pairing channels. Here, we dynamically drive an asymmetric oscillation of the gap to study the effect on the Higgs spectrum.

Our approach is purely phenomenological and neglects additional polarization dependencies which are likely to occur due to the asymmetric driving. As the polarization dependence is hard to predict without an in detail understanding of the actual microscopic coupling, we neglect it for our approach.

Using the modified pseudomagnetic field and performing a linearization in the same way as in Sec. III one would neglect important contributions from the products between the asymmetric driving term and the deviations, e.g., $\delta \sin ^{2}(\Omega t) f_{k}^{A} x_{k}(t)$, etc. Therefore we solve the Bloch equations for this section numerically without any approximation by integrating the differential equations in time. As we haven't added an additional polarization dependence, we can suppress the CDF contribution by choosing $\theta=\pi / 4$ to obtain the contribution from the Higgs channel.

In Fig. 7, we show the temperature dependence of the THG intensity for a $d$-wave gap with an asymmetric driving $f_{k}^{A}=$ 1 , i.e., a distortion in the $s$-wave channel. This corresponds to driving the $B_{1 g}$ mode of the $d$-wave gap. It does not cor-

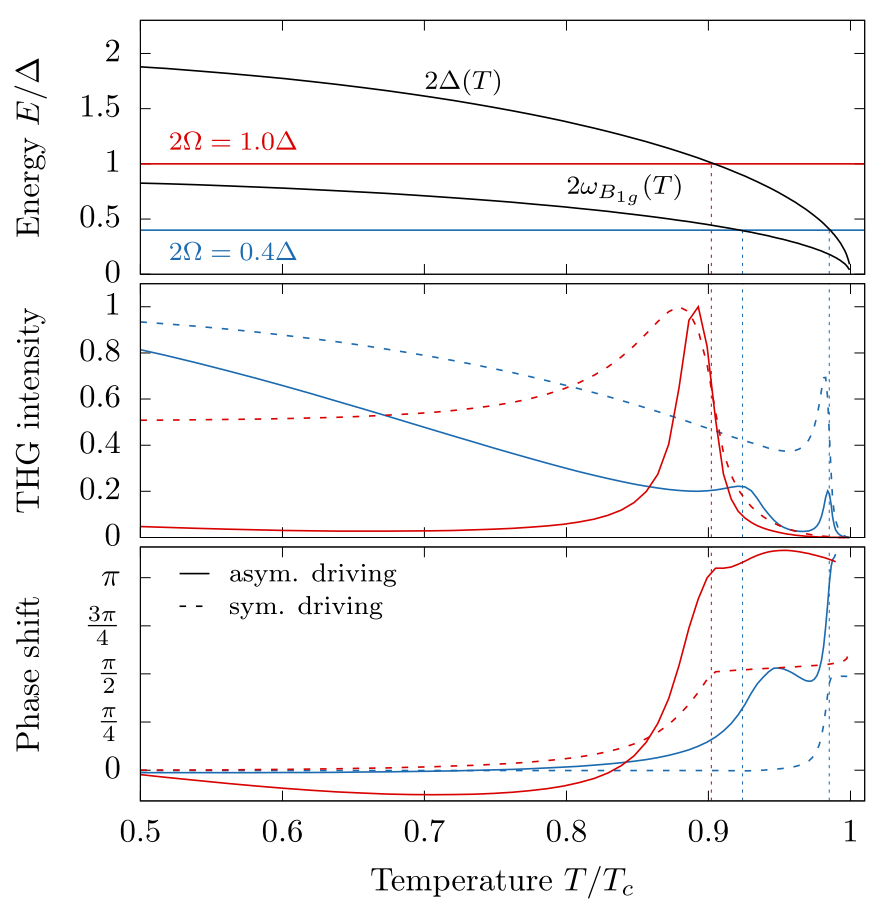

FIG. 7. Temperature dependence of THG for $d$ wave for two different driving frequencies using the asymmetric driving scheme (84) with $\delta_{A}=0.1$. The top row shows the driving frequencies in relation to the energy gap and the second mode. The middle row shows the THG intensity and the bottom row the phase. The result from the asymmetric driving (solid) is compared with the standard driving (dashed).

responds directly to any experimental excitation scheme, but acts as a proof of principle to study the induced effects. Any possible experimental realizations are likely a superposition of fundamental symmetries. For low driving frequencies, one can observe a second resonance peak in the THG signal below the $2 \Delta$ peak, which is also accompanied by a phase change of $\pi / 2$. Higher driving frequencies do not show such a resonance. This can be understood better, by calculating the frequency dependence of THG for different temperatures from which one can extract the temperature dependence of the modes. For the chosen set of parameters, the resulting curve of the second mode follows approximately $\omega_{B_{1 g}}(T)=0.44 \Delta(T)$ which is also shown in the upper row of Fig. 7. As one can see, the chosen higher driving frequency of $2 \Omega=\Delta$ stays always above the second mode which explains that there is no second resonance peak. For the lower driving frequency of $2 \Omega=0.4 \Delta$, the second resonance peak appears at the point where $2 \Omega=\omega_{B_{1 g}}(T)$

In a recent THG experiments on cuprates, a collective mode below $2 \Delta$ was observed [52]. An asymmetric driving and resonantly excitation of a $B_{1 g}$ mode (or other non- $A_{1 g}$ modes) could be, in principle, an explanation of additional modes.

\section{SUMMARY AND DISCUSSION}

In this work, we analyze the induced gap oscillations in unconventional superconductors due to periodic driving with 
THz light within BCS theory in the Anderson pseudospin formalism. We show exemplary for $d$-wave how a non- $s$ wave symmetry broadens both the resonance peak and the phase change in the oscillation amplitude and derive analytic expressions for the oscillations. The gap oscillations lead to third-harmonic generation whose intensity shows a resonance peak if twice the driving frequency coincidences with the energy of the Higgs mode. Although, the calculated spectra for pure $s$ and $d$ wave differ significantly, experimental conditions can wash out the spectrum for $s$ wave as well, which makes a differentiation of the two gap symmetries only from an observation of the spectrum difficult. We compare contributions from CDF and Higgs and illustrated that the Higgs contribution to THG follows closely the amplitude of the gap oscillation, whereas the CDF contribution shows still a sharp peak even in the non- $s$-wave case. The temperature dependence of the THG signal as measured in the experiment shows the same characteristics as the THG signal as a function of the driving frequency.

In addition one finds that more complex gap symmetries, shown exemplary for $d+s$ wave, which contain multiple local gap maxima show resonances for each maxima. Hereby it is the contribution of the Higgs oscillation which shows this feature as the composite gap contains two Higgs modes with frequencies at these local gap maxima. Thus THG experiments can be used to detect the occurrence of multiple Higgs modes in unconventional superconductors.

For $d$ wave, or in general for gap symmetries where the squared symmetry function is isotropic in $x$ and $y$ directions, the polarization dependence of the THG signal is the same as for $s$ wave. For a simple square-lattice tight-binding dispersion, the Higgs contribution is polarization-independent, whereas the CDF contribution has a characteristic dependency. However, deviations both from the isotropy of the gap symmetry as well as the simple tight-binding dispersion lead also to deviations in the polarization dependence and the Higgs contribution can become polarization dependent. Yet, if the band-dispersion is known, a measurement of the polarization dependency allows to distinguish the individual contributions from CDF and Higgs. This result is probably true even beyond a clean-limit analysis. Typical impurityscattering introduces no additional direction dependence as the scattered momenta are randomly distributed. Therefore the polarization dependence should be unaffected [51,59].

In our analysis for the clean limit, where the coupling to the vector potential is exclusively through the nonparabolicity of the band dispersion, the CDF contribution exceeds in general the Higgs contribution. Nevertheless, this work gives an interesting insight into the driven dynamics of unconventional superconductors. On the one hand, the Higgs contribution can still show its unique features as seen in the $d+s$-wave case with the two-peak structure, which is a result from the Higgs contribution alone. On the other hand, as it was shown elsewhere [56-60], effects beyond BCS theory and impurity scattering in the dirty-limit can strongly enhance the Higgs contribution over the CDF contribution.

Finally, we propose an asymmetric driving scheme to model experiments where the coupling of the driving field acts nonsymmetrically with respect to the ground-state symmetry on the condensate. Such asymmetry induces asymmetric oscillations of the condensate which can show up as an additional oscillation frequency of the gap, dependent on the symmetry of the gap and the deviation. With our proof of principle calculation we show that THG experiments should be, in principle, able to measure asymmetric Higgs modes and provide therefore the same information as pump-probe experiments. However, as the proposed phenomenological asymmetric driving scheme may be difficult to realize experimentally, the information obtained by THG experiments are limited in this respect.

In addition to the measurement of the collective modes itself, spectroscopy of Higgs modes may be used in future as an alternative or complementary probe to investigate the gap symmetry. A controlled excitation and observation of Higgs modes allows to gain information about symmetry properties of the underlying gap [42]. In a more general sense, THG experiments may also serve as a new measure for defining superconductivity in nonequilibrium. Recent experiments and theoretical studies on light-induced superconductivity [43-48] raise the question on how one defines superconductivity in a short-lived nonequilibrium state. So far, the criteria only include the vanishing resistivity property of superconductors measured by a divergent imaginary part of the optical conductivity for $\omega \rightarrow 0$, however the expelling of a magnetic field, i.e., the Meissner effect, has not yet been considered. As the Meissner effect is induced by the Anderson-Higgs mechanism, a measurement of the Higgs mode should be an equivalent fingerprint of superconductivity. While the repulsion of a magnetic field on an ultrashort timescale is difficult to measure or even impossible, a resonant behavior of the THG signal in the light-induced superconducting state could potentially be realized.

In addition to pump-probe experiments, where the gap is quenched by a short pulse and the following intrinsic Higgs oscillations can be observed, THG experiments can serve as an alternative tool for identifying Higgs modes of a superconductor. These driven experiments have some advantages over the pump-probe experiments. No ultrashort single-cycle pulses are required and the strong-damping of Higgs modes in gaps with nodes are partly overcome due to the forced periodic driving and oscillation of the gap. Thus, in the context of Higgs spectroscopy, i.e., the detection and characterization of Higgs modes in superconductors, THG experiments extend the range of possible experimental setups.

\section{ACKNOWLEDGMENTS}

We thank B. Fauseweh, A. Schnyder, and N. Tsuji as well as S. Kaiser, H. Chu, M. J. Kim, and R. Shimano for helpful discussions both from theory and experimental side. We also thank the Max Planck-UBC-UTokyo Center for Quantum Materials for fruitful collaborations and financial support.

\section{APPENDIX A: EXPANSION OF DRIVING TERM}

We assume that the $x$ and $y$ directions are equivalent for $f_{k}^{2}$ and $\epsilon_{k}$, i.e., $f\left(k_{x}, k_{y}\right)^{2}=f\left(k_{y}, k_{x}\right)^{2}$ and $\epsilon\left(k_{x}, k_{y}\right)=\epsilon\left(k_{y}, k_{x}\right)$. It follows for any function $a_{k}$ whose $\boldsymbol{k}$ dependence stems only 
from $f_{k}^{2}$ and $\epsilon_{k}$ that

$$
\sum_{k}\left(\partial_{x x}^{2} \epsilon_{k}\right) a_{k}=\sum_{k}\left(\partial_{y y}^{2} \epsilon_{k}\right) a_{k}=\frac{1}{2} \sum_{k}\left(\nabla^{2} \epsilon_{k}\right) a_{k} .
$$

This assumption is true for functions like $\epsilon_{k}=\epsilon(|\boldsymbol{k}|)$ or $\epsilon_{k} \propto$ $\cos k_{x}+\cos k_{y}$ and $f_{k}=1$ or $f_{k} \propto \cos k_{x}-\cos k_{y}$. As we are mostly interested in values close to the Fermi energy $\epsilon_{\mathrm{F}}$, i.e., values of $\epsilon_{k} \approx 0$, we expand the Laplacian of the dispersion to first order in the dispersion

$$
\frac{1}{2} \nabla^{2} \epsilon_{k}=\alpha_{0}+\alpha_{1} \epsilon_{k}+\mathcal{O}\left(\epsilon_{k}^{2}\right) .
$$

As an example, for the single-band tight-binding dispersion on the square lattice with nearest neighbor hopping $t$

$$
\epsilon_{k}=-2 t\left(\cos k_{x}+\cos k_{y}\right)-\epsilon_{\mathrm{F}}
$$

it follows

$$
\frac{1}{2} \nabla^{2} \epsilon_{k}=t\left(\cos k_{x}+\cos k_{y}\right)=-\frac{\epsilon_{\mathrm{F}}}{2}-\frac{\epsilon_{k}}{2}
$$

and the expansion to first order with $\alpha_{0}=-\epsilon_{\mathrm{F}} / 2$ and $\alpha_{1}=$ $-1 / 2$ becomes exact. Further we assume that the mixed derivatives of the dispersion vanishes, i.e., $\partial_{x y}^{2} \epsilon_{k}=0$.

\section{APPENDIX B: INVERSE LAPLACE TRANSFORM}

The inverse Laplace transform for $I_{1}(s)$ in Eq. (46) is trivial and we get

$$
I_{1}(t)=\frac{1-\cos (2 \Omega t)}{4} .
$$

For the second term $I_{2}(t)$ in Eq. (47), the Bromwich integral has to be explicitly evaluated

$$
I_{2}(t)=\frac{1}{2 \pi i} \int_{\gamma-i \infty}^{\gamma+i \infty} e^{s t} I_{2}(s) d s
$$

The integrand has three poles at $s=0, \pm 2 i \Omega$ and depending on $f \in[-1,1]$ a continuous line of branchpoints between $s=-2 i \Delta$ and $s=2 i \Delta$. We evaluate the integral by extending the path into the complex plane considering the contour in Fig. 8, which is chosen such that the poles contribute with their residue and a branch cut between $s=-2 i \Delta$ and $s=2 i \Delta$ on the imaginary axis is excluded. The closed loop integral vanishes as no poles are included, the outer integral $c_{1}$ vanish if the radius goes to infinity. The integral denoted by $c_{2}$, i.e., the paths from infinity to the poles and between the poles and the end of the branch cut along the imaginary axis cancel each other. We are left with the contributions around the poles $c_{3}, c_{4}, c_{5}$ and the paths left and right of of the branch cut $c_{6}$ and $c_{7}$

$$
\int_{\gamma-i \infty}^{\gamma+i \infty}=-\int_{c_{3}}-\int_{c_{4}}-\int_{c_{5}}-\int_{c_{6}}-\int_{c_{7}}
$$

Each of the small circles around the poles contribute with their residue in the limit of the radius going to zero

$$
\sum_{n=3,4,5} \frac{1}{2 \pi i} \int_{c_{n}} e^{s t} I_{2}(s) d s=\sum_{r=0, \pm 2 i \Omega} \operatorname{Res}_{r}\left(e^{s t} I_{2}(s)\right) .
$$

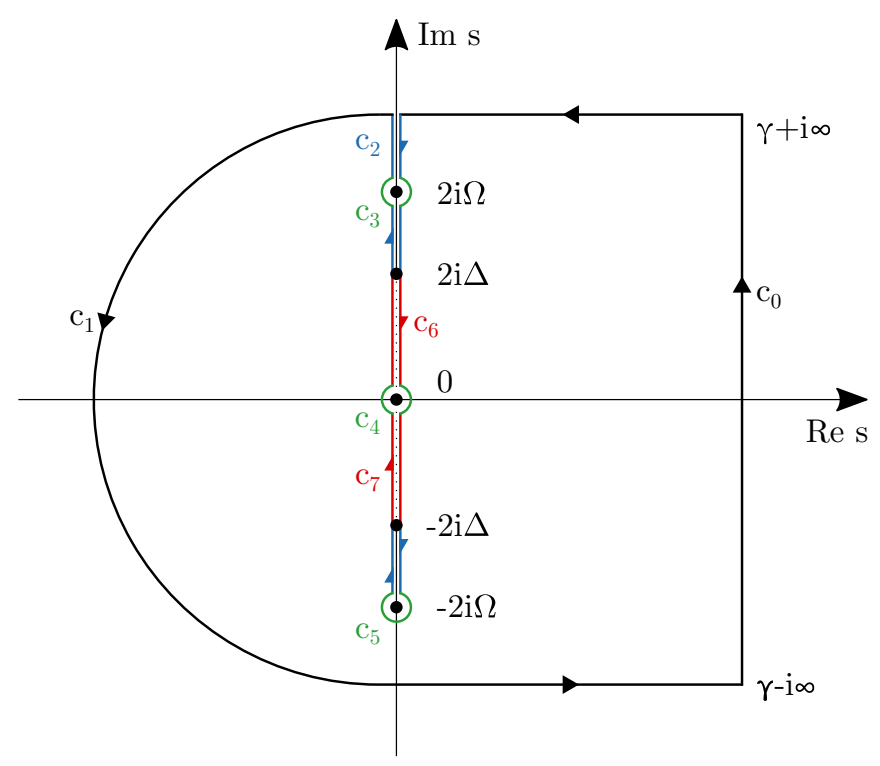

FIG. 8. Poles at $s=0, \pm 2 i \Omega$ and continuous line of branch points at $s=2 i \Delta f$ of the integrand in Eq. (B2). The Bromwich integral $c_{0}$ is extended to the shown contour in the complex plane, where the residues of the poles $c_{3}, c_{4}, c_{5}$ and the paths $c_{6}, c_{7}$ next to the branch cut contribute.

For the residues, it follows

$$
\begin{gathered}
\operatorname{Res}_{0}\left(e^{s t} I_{2}(s)\right)=\frac{1}{4 \lambda \int f^{2} d \varphi}, \\
\operatorname{Res}_{ \pm 2 i \Omega}\left(e^{s t} I_{2}(s)\right)=-\frac{\Omega}{8} e^{ \pm 2 i \Omega t} \\
\times \frac{1}{\lambda \int d \varphi f^{2} \sqrt{\Delta^{2} f^{2}-\Omega^{2}} \sin ^{-1}\left(\frac{\Omega}{\Delta|f|}\right)} .
\end{gathered}
$$

The integrals along $c_{6}$ and $c_{7}$ can be parametrized with $s(r)=$ $\epsilon+i r$ with $r \in[2 \Delta,-2 \Delta]$ for $c_{6}$ and $r \in[-2 \Delta, 2 \Delta]$ for $c_{7}$ for $\epsilon \rightarrow 0$. We obtain

$$
\begin{aligned}
\frac{1}{2 \pi i} \int_{c_{4} / c_{6}} e^{s t} I_{2}(s) d s \\
=\frac{ \pm 1}{2 \pi i} \int_{-2 \Delta}^{2 \Delta} d r \frac{\Omega^{2}}{4 \Omega^{2}-r^{2}} \\
\quad \times \frac{e^{ \pm i r t}}{\lambda \int d \varphi f^{2} \sqrt{4 \Delta^{2} f^{2}-r^{2}} \sin ^{-1}\left(\frac{r}{2 \Delta|f|}\right)} .
\end{aligned}
$$

\section{APPENDIX C: DERIVATION OF THG}

The Fourier transform of Eq. (69) reads

$$
\begin{aligned}
j_{\| \perp}^{(3)}(\omega)= & -2 e^{2} \sum_{k} D_{\epsilon_{k}}^{\| \perp}(\theta) \\
& \times \frac{1}{\sqrt{2 \pi}} \int A_{0}\left(\omega^{\prime}\right) z_{k}\left(\omega-\omega^{\prime}\right) d \omega^{\prime} .
\end{aligned}
$$


The expression for the vector potential (4) is inserted and the convolution at $\omega=3 \Omega$ is evaluated

$$
j_{\| \perp}^{(3)}(3 \Omega)=i e^{2} A_{0} \sum_{k} D_{\epsilon_{k}}^{\| \perp}(\theta) z_{k}(s=2 i \Omega)
$$

We make use of the solution $z_{k}(s=2 i \Omega)$ from the linearized Bloch equations (35) including also the temperature dependence. Using the definitions

$$
\begin{aligned}
& x_{0}(s)=V \sum_{k} \frac{f_{k}^{2}}{E_{k}\left(4 E_{k}^{2}+s^{2}\right)} \tanh \left(\frac{E_{k}}{2 k_{B} T}\right), \\
& x_{1}(s)=V \sum_{k} \frac{f_{k}^{4}}{E_{k}\left(4 E_{k}^{2}+s^{2}\right)} \tanh \left(\frac{E_{k}}{2 k_{B} T}\right), \\
& x_{2}(s)=V \sum_{k} \frac{\epsilon_{k} f_{k}^{2}}{E_{k}\left(4 E_{k}^{2}+s^{2}\right)} \tanh \left(\frac{E_{k}}{2 k_{B} T}\right), \\
& x_{3}(s)=V \sum_{k} \frac{\epsilon_{k}^{2} f_{k}^{2}}{E_{k}\left(4 E_{k}^{2}+s^{2}\right)} \tanh \left(\frac{E_{k}}{2 k_{B} T}\right), \\
& x_{4}^{\| \perp}(s)=V \sum_{k} \frac{D_{\epsilon_{k}}^{\| \perp} f_{k}^{2}}{E_{k}\left(4 E_{k}^{2}+s^{2}\right)} \tanh \left(\frac{E_{k}}{2 k_{B} T}\right), \\
& x_{5}^{\| \perp}(s)=V \sum_{k} \frac{\epsilon_{k} D_{\epsilon_{k}}^{\| \perp} f_{k}^{2}}{E_{k}\left(4 E_{k}^{2}+s^{2}\right)} \tanh \left(\frac{E_{k}}{2 k_{B} T}\right), \\
& x_{6}^{\| \perp}(s)=V \sum_{k} \frac{D_{\epsilon_{k}}^{\|} D_{\epsilon_{k}}^{\| \perp} f_{k}^{2}}{E_{k}\left(4 E_{k}^{2}+s^{2}\right)} \tanh \left(\frac{E_{k}}{2 k_{B} T}\right)
\end{aligned}
$$

one obtains

$$
\begin{gathered}
j_{\| \perp}^{(3) \mathrm{H}}(3 \Omega) \propto 2 \Delta x_{5}^{\| \perp}(2 i \Omega) \delta \Delta^{\prime}(2 i \Omega), \\
j_{\| \perp}^{(3) \mathrm{P}}(3 \Omega) \propto-s \Delta x_{4}^{\| \perp}(2 i \Omega) \delta \Delta^{\prime \prime}(2 i \Omega), \\
j_{\| \perp}^{(3) \mathrm{CDF}}(3 \Omega) \propto-\Delta^{2} e^{2} A_{0}^{2}(2 i \Omega) x_{6}^{\| \perp}(2 i \Omega)
\end{gathered}
$$

where the real and imaginary parts of the gap can be written as

$$
\begin{aligned}
& \delta \Delta^{\prime}(s) \\
& \quad=\Delta e^{2} A_{0}^{2}(s) \\
& \quad \times \frac{s^{2} x_{2}(s) x_{4}^{\|}(s)+2 x_{5}^{\|}(s)\left(2 \Delta^{2} x_{1}(s)+2 x_{3}(s)-1\right)}{2 s^{2} x_{2}(s)^{2}+2\left(2 x_{3}(s)-1\right)\left(2 \Delta^{2} x_{1}(s)+2 x_{3}(s)-1\right)}
\end{aligned}
$$

and

$$
\begin{aligned}
& \delta \Delta^{\prime \prime}(s) \\
& =s \Delta e^{2} A_{0}^{2}(s) \\
& \quad \times \frac{2 x_{3}(s) x_{4}^{\|}(s)-x_{4}^{\|}(s)-2 x_{2}(s) x_{5}^{\|}(s)}{2 s^{2} x_{2}(s)^{2}+2\left(2 x_{3}(s)-1\right)\left(2 \Delta^{2} x_{1}(s)+2 x_{3}(s)-1\right)} .
\end{aligned}
$$

To understand these expression, we make use of the same approximations as in the previous sections. It follows

$$
\begin{aligned}
& x_{2}(s)=0, \\
& x_{4}^{\perp}(s)=0, \\
& x_{5}^{\perp}(s)=0, \\
& x_{4}^{\|}(s)=V \sum_{\boldsymbol{k}} \frac{\partial_{x x}^{2} \epsilon_{k} f_{k}^{2}}{E_{k}\left(4 E_{k}^{2}+s^{2}\right)} \tanh \left(\frac{E_{k}}{2 k_{B} T}\right)=\alpha_{0} x_{0}(s) \text {, } \\
& x_{5}^{\|}(s)=V \sum_{k} \frac{\epsilon_{k} \partial_{x x}^{2} \epsilon_{k} f_{k}^{2}}{E_{k}\left(4 E_{k}^{2}+s^{2}\right)} \tanh \left(\frac{E_{k}}{2 k_{B} T}\right) \\
& =\alpha_{1} x_{3}(s) \text {, } \\
& x_{6}^{\perp}(s)=\frac{1}{4} \sin 4 \theta \\
& \times V \sum_{k} \frac{\left(\partial_{x x}^{2} \epsilon_{k}\right)^{2} f_{k}^{2}}{E_{k}\left(4 E_{k}^{2}+s^{2}\right)} \tanh \left(\frac{E_{k}}{2 k_{B} T}\right)-\frac{1}{4} \sin 4 \theta \\
& \times V \sum_{k} \frac{\left(\partial_{x x}^{2} \epsilon_{k}\right)\left(\partial_{y y}^{2} \epsilon_{k}\right) f_{k}^{2}}{E_{k}\left(4 E_{k}^{2}+s^{2}\right)} \tanh \left(\frac{E_{k}}{2 k_{B} T}\right) \\
& \approx \frac{1}{4} \sin 4 \theta\left(2 \alpha_{0}^{2} x_{0}(s)+2 \alpha_{1}^{2} x_{3}(s)\right), \\
& x_{6}^{\|}(s)=\left(1-\frac{1}{2} \sin ^{2} 2 \theta\right) \\
& \times V \sum_{k} \frac{\left(\partial_{x x}^{2} \epsilon_{k}\right)^{2} f_{k}^{2}}{E_{k}\left(4 E_{k}^{2}+s^{2}\right)} \tanh \left(\frac{E_{k}}{2 k_{B} T}\right)+\frac{1}{2} \sin ^{2} 2 \theta \\
& \times V \sum_{k} \frac{\left(\partial_{x x}^{2} \epsilon_{k}\right)\left(\partial_{y y}^{2} \epsilon_{k}\right) f_{k}^{2}}{E_{k}\left(4 E_{k}^{2}+s^{2}\right)} \tanh \left(\frac{E_{k}}{2 k_{B} T}\right) \\
& \approx\left(1-\frac{1}{2} \sin ^{2} 2 \theta\right)\left(2 \alpha_{0}^{2} x_{0}(s)+2 \alpha_{1}^{2} x_{3}(s)\right) \text {. }
\end{aligned}
$$

where we identified $\sin ^{4} \theta+\cos ^{4} \theta=1-\frac{1}{2} \sin ^{2} 2 \theta$, $2 \sin ^{2} \theta \cos ^{2} \theta=\frac{1}{2} \sin ^{2} 2 \theta$, and $\sin \theta \cos \theta\left(\cos ^{2} \theta-\sin ^{2} \theta\right)=$ $\frac{1}{4} \sin 4 \theta$ and neglected sums with terms $\propto\left(\partial_{x x}^{2} \epsilon_{k}\right)\left(\partial_{y y}^{2} \epsilon_{k}\right)$. We use the general fact that $x_{3}(s)=\frac{1}{2}-\Delta^{2} x_{1}(s)-\frac{1}{4} s^{2} x_{0}(s)$ and the expression for the real part of the gap reduces to the result in Eq. (41) from the previous section

$$
\delta \Delta^{\prime}(s)=\frac{1}{2} \alpha_{1} \Delta e^{2} A_{0}^{2}(s)\left(1+\frac{1}{2 x_{3}(s)-1}\right),
$$

whereas the imaginary part reduces to Eq. (55)

$$
\delta \Delta^{\prime \prime}(s)=-\alpha_{0} \Delta e^{2} \frac{A_{0}^{2}(s)}{s} .
$$

Writing the sums as integrals using $x_{3}(s)=1 / 2-H(s) / 2$ and $x_{0}(s)=2 G(s)$, one obtains the result Eqs. (75)-(78). 
[1] J. Orenstein, Ultrafast spectroscopy of quantum materials, Phys. Today 65(9), 44 (2012).

[2] D. Nicoletti and A. Cavalleri, Nonlinear light-matter interaction at terahertz frequencies, Adv. Opt. Photon. 8, 401 (2016).

[3] C. Giannetti, M. Capone, D. Fausti, M. Fabrizio, F. Parmigiani, and D. Mihailovic, Ultrafast optical spectroscopy of strongly correlated materials and high-temperature superconductors: A non-equilibrium approach, Adv. Phys. 65, 58 (2016).

[4] P. W. Higgs, Broken Symmetries and the Masses of Gauge Bosons, Phys. Rev. Lett. 13, 508 (1964).

[5] C. Varma, Higgs boson in superconductors, J. Low. Temp. Phys. 126, 901 (2002).

[6] D. Podolsky, A. Auerbach, and D. P. Arovas, Visibility of the amplitude (Higgs) mode in condensed matter, Phys. Rev. B 84, 174522 (2011).

[7] Y. Barlas and C. M. Varma, Amplitude or Higgs modes in $d$-wave superconductors, Phys. Rev. B 87, 054503 (2013).

[8] A. Pashkin and A. Leitenstorfer, Particle physics in a superconductor, Science 345, 1121 (2014).

[9] D. Pekker and C. Varma, Amplitude/higgs modes in condensed matter physics, Annu. Rev. Condens. Matter Phys 6, 269 (2015).

[10] P. W. Anderson, Coherent excited states in the theory of superconductivity: Gauge invariance and the meissner effect, Phys. Rev. 110, 827 (1958).

[11] R. Sooryakumar and M. V. Klein, Raman Scattering by Superconducting-Gap Excitations and Their Coupling to Charge-Density Waves, Phys. Rev. Lett. 45, 660 (1980).

[12] P. B. Littlewood and C. M. Varma, Gauge-Invariant Theory of the Dynamical Interaction of Charge Density Waves and Superconductivity, Phys. Rev. Lett. 47, 811 (1981).

[13] P. B. Littlewood and C. M. Varma, Amplitude collective modes in superconductors and their coupling to charge-density waves, Phys. Rev. B 26, 4883 (1982).

[14] M.-A. Méasson, Y. Gallais, M. Cazayous, B. Clair, P. Rodière, L. Cario, and A. Sacuto, Amplitude Higgs mode in the $2 \mathrm{H}-$ $\mathrm{NbSe}_{2}$ superconductor, Phys. Rev. B 89, 060503(R) (2014).

[15] R. Grasset, T. Cea, Y. Gallais, M. Cazayous, A. Sacuto, L. Cario, L. Benfatto, and M.-A. Méasson, Higgs-mode radiance and charge-density-wave order in $2 \mathrm{H}-\mathrm{NbSe}_{2}$, Phys. Rev. B 97, 094502 (2018).

[16] R. Grasset, Y. Gallais, A. Sacuto, M. Cazayous, S. MañasValero, E. Coronado, and M.-A. Méasson, Pressure-Induced Collapse of the Charge Density Wave and Higgs Mode Visibility in 2H-TaS 2 , Phys. Rev. Lett. 122, 127001 (2019).

[17] R. Matsunaga and R. Shimano, Nonequilibrium BCS State Dynamics Induced by Intense Terahertz Pulses in a Superconducting NbN Film, Phys. Rev. Lett. 109, 187002 (2012).

[18] R. Matsunaga, Y. I. Hamada, K. Makise, Y. Uzawa, H. Terai, Z. Wang, and R. Shimano, Higgs Amplitude Mode in the BCS Superconductors $\mathrm{Nb}_{1-x} \mathrm{Ti}_{x} \mathbf{N}$ Induced by Terahertz Pulse Excitation, Phys. Rev. Lett. 111, 057002 (2013).

[19] B. Mansart, J. Lorenzana, A. Mann, A. Odeh, M. Scarongella, M. Chergui, and F. Carbone, Coupling of a high-energy excitation to superconducting quasiparticles in a cuprate from coherent charge fluctuation spectroscopy, Proc. Natl. Acad. Sci. USA 110, 4539 (2013).

[20] K. Katsumi, N. Tsuji, Y. I. Hamada, R. Matsunaga, J. Schneeloch, R. D. Zhong, G. D. Gu, H. Aoki, Y. Gallais, and R. Shimano, Higgs Mode in the $d$-Wave Superconductor
$\mathrm{Bi}_{2} \mathrm{Sr}_{2} \mathrm{CaCu}_{2} \mathrm{O}_{8+x}$ Driven by an Intense Terahertz Pulse, Phys. Rev. Lett. 120, 117001 (2018).

[21] A. F. Kemper, M. A. Sentef, B. Moritz, J. K. Freericks, and T. P. Devereaux, Direct observation of Higgs mode oscillations in the pump-probe photoemission spectra of electron-phonon mediated superconductors, Phys. Rev. B 92, 224517 (2015).

[22] B. Nosarzewski, B. Moritz, J. K. Freericks, A. F. Kemper, and T. P. Devereaux, Amplitude mode oscillations in pump-probe photoemission spectra from a $d$-wave superconductor, Phys. Rev. B 96, 184518 (2017).

[23] L. Schwarz, B. Fauseweh, and D. Manske, Momentum-resolved analysis of condensate dynamic and Higgs oscillations in quenched superconductors with tr-ARPES, arXiv:2002.05904.

[24] A. F. Volkov and S. M. Kogan, Collisionless relaxation of the energy gap in superconductors, Sov. Phys. JETP 38, 1018 (1974).

[25] R. A. Barankov, L. S. Levitov, and B. Z. Spivak, Collective Rabi Oscillations and Solitons in a Time-Dependent BCS Pairing Problem, Phys. Rev. Lett. 93, 160401 (2004).

[26] E. A. Yuzbashyan, O. Tsyplyatyev, and B. L. Altshuler, Relaxation and Persistent Oscillations of the Order Parameter in Fermionic Condensates, Phys. Rev. Lett. 96, 097005 (2006).

[27] R. A. Barankov and L. S. Levitov, Synchronization in the BCS Pairing Dynamics as a Critical Phenomenon, Phys. Rev. Lett. 96, 230403 (2006).

[28] E. A. Yuzbashyan and M. Dzero, Dynamical Vanishing of the Order Parameter in a Fermionic Condensate, Phys. Rev. Lett. 96, 230404 (2006).

[29] T. Papenkort, V. M. Axt, and T. Kuhn, Coherent dynamics and pump-probe spectra of BCS superconductors, Phys. Rev. B 76, 224522 (2007).

[30] J. Unterhinninghofen, D. Manske, and A. Knorr, Theory of ultrafast nonequilibrium dynamics in $d$-wave superconductors, Phys. Rev. B 77, 180509(R) (2008).

[31] T. Papenkort, T. Kuhn, and V. M. Axt, Coherent control of the gap dynamics of bcs superconductors in the nonadiabatic regime, Phys. Rev. B 78, 132505 (2008).

[32] Y.-Z. Chou, Y. Liao, and M. S. Foster, Twisting Anderson pseudospins with light: Quench dynamics in terahertz-pumped BCS superconductors, Phys. Rev. B 95, 104507 (2017).

[33] A. P. Schnyder, D. Manske, and A. Avella, Resonant generation of coherent phonons in a superconductor by ultrafast optical pump pulses, Phys. Rev. B 84, 214513 (2011).

[34] H. Krull, D. Manske, G. S. Uhrig, and A. P. Schnyder, Signatures of nonadiabatic BCS state dynamics in pump-probe conductivity, Phys. Rev. B 90, 014515 (2014).

[35] M. Zachmann, M. D. Croitoru, A. Vagov, V. M. Axt, T. Papenkort, and T. Kuhn, Ultrafast terahertz-field-induced dynamics of superconducting bulk and quasi-1D samples, New J. Phys. 15, 055016 (2013).

[36] A. Akbari, A. P. Schnyder, D. Manske, and I. Eremin, Theory of nonequilibrium dynamics of multiband superconductors, Europhys. Lett. 101, 17002 (2013).

[37] H. Krull, N. Bittner, G. S. Uhrig, D. Manske, and A. P. Schnyder, Coupling of Higgs and Leggett modes in non-equilibrium superconductors, Nat. Commun. 7, 11921 (2016).

[38] Y. Murakami, P. Werner, N. Tsuji, and H. Aoki, Multiple amplitude modes in strongly coupled phonon-mediated superconductors, Phys. Rev. B 93, 094509 (2016). 
[39] A. Moor, A. F. Volkov, and K. B. Efetov, Amplitude Higgs Mode and Admittance in Superconductors with a Moving Condensate, Phys. Rev. Lett. 118, 047001 (2017).

[40] S. Nakamura, Y. Iida, Y. Murotani, R. Matsunaga, H. Terai, and R. Shimano, Infrared Activation of the Higgs Mode by Supercurrent Injection in Superconducting NbN, Phys. Rev. Lett. 122, 257001 (2019).

[41] F. Peronaci, M. Schiró, and M. Capone, Transient Dynamics of $d$-Wave Superconductors after a Sudden Excitation, Phys. Rev. Lett. 115, 257001 (2015).

[42] L. Schwarz, B. Fauseweh, N. Tsuji, N. Cheng, N. Bittner, H. Krull, M. Berciu, G. S. Uhrig, A. P. Schnyder, S. Kaiser, and D. Manske, Classification and characterization of nonequilibrium Higgs modes in unconventional superconductors, Nat. Commun. 11, 287 (2020).

[43] D. Fausti, R. I. Tobey, N. Dean, S. Kaiser, A. Dienst, M. C. Hoffmann, S. Pyon, T. Takayama, H. Takagi, and A. Cavalleri, Light-induced superconductivity in a stripe-ordered cuprate, Science 331, 189 (2011).

[44] S. Kaiser, C. R. Hunt, D. Nicoletti, W. Hu, I. Gierz, H. Y. Liu, M. Le Tacon, T. Loew, D. Haug, B. Keimer, and A. Cavalleri, Optically induced coherent transport far above $T_{c}$ in underdoped $\mathrm{YBa}_{2} \mathrm{Cu}_{3} \mathrm{O}_{6+\delta}$, Phys. Rev. B 89, 184516 (2014).

[45] M. Mitrano, A. Cantaluppi, D. Nicoletti, S. Kaiser, A. Perucchi, S. Lupi, P. Di Pietro, D. Pontiroli, M. Riccò, S. R. Clark, D. Jaksch, and A. Cavalleri, Possible light-induced superconductivity in K3C60 at high temperature, Nature (London) 530, 461 (2016).

[46] N. Bittner, T. Tohyama, S. Kaiser, and D. Manske, Possible light-induced superconductivity in a strongly correlated electron system, J. Phys. Soc. Jpn. 88, 044704 (2019).

[47] S. Paeckel, B. Fauseweh, A. Osterkorn, T. Köhler, D. Manske, and S. Manmana, Detecting superconductivity out-ofequilibrium, Phys. Rev. B 101, 180507(R) (2020).

[48] M. Buzzi, G. Jotzu, A. Cavalleri, J. I. Cirac, E. A. Demler, B. I. Halperin, M. D. Lukin, T. Shi, Y. Wang,, and D. Podolsky, Higgs-mediated optical amplification in a non-equilibrium superconductor, arXiv:1908.10879.

[49] N. Tsuji and H. Aoki, Theory of Anderson pseudospin resonance with Higgs mode in superconductors, Phys. Rev. B 92, 064508 (2015).

[50] R. Matsunaga, N. Tsuji, H. Fujita, A. Sugioka, K. Makise, Y. Uzawa, H. Terai, Z. Wang, H. Aoki, and R. Shimano, Lightinduced collective pseudospin precession resonating with Higgs mode in a superconductor, Science 345, 1145 (2014).

[51] R. Matsunaga, N. Tsuji, K. Makise, H. Terai, H. Aoki, and R. Shimano, Polarization-resolved terahertz third-harmonic generation in a single-crystal superconductor $\mathrm{NbN}$ : Dominance of the Higgs mode beyond the BCS approximation, Phys. Rev. B 96, 020505(R) (2017).

[52] H. Chu, M.-J. Kim, K. Katsumi, S. Kovalev, R. D. Dawson, L. Schwarz, N. Yoshikawa, G. Kim, D. Putzky, Z. Z. Li, H. Raffy, S. Germanskiy, J.-C. Deinert, N. Awari, I. Ilyakov, B.
Green, M. Chen, M. Bawatna, G. Christiani, G. Logvenov, Y. Gallais, A. V. Boris, B. Keimer, A. P. Schnyder, D. Manske, M. Gensch, Z. Wang, R. Shimano, and S. Kaiser, Phase-resolved Higgs response in superconducting cuprates, Nat. Commun. 11, 1793 (2020).

[53] T. Cea, C. Castellani, and L. Benfatto, Nonlinear optical effects and third-harmonic generation in superconductors: Cooper pairs versus Higgs mode contribution, Phys. Rev. B 93, 180507(R) (2016).

[54] N. Tsuji, Y. Murakami, and H. Aoki, Nonlinear light-Higgs coupling in superconductors beyond BCS: Effects of the retarded phonon-mediated interaction, Phys. Rev. B 94, 224519 (2016).

[55] T. Cea, P. Barone, C. Castellani, and L. Benfatto, Polarization dependence of the third-harmonic generation in multiband superconductors, Phys. Rev. B 97, 094516 (2018).

[56] T. Jujo, Two-photon absorption by impurity scattering and amplitude mode in conventional superconductors, J. Phys. Soc. Jpn. 84, 114711 (2015).

[57] T. Jujo, Quasiclassical theory on third-harmonic generation in conventional superconductors with paramagnetic impurities, J. Phys. Soc. Jpn. 87, 024704 (2018).

[58] T. Yu and M. W. Wu, Gauge-invariant theory of quasiparticle and condensate dynamics in response to terahertz optical pulses in superconducting semiconductor quantum wells. I. s-wave superconductivity in the weak spin-orbit coupling limit, Phys. Rev. B 96, 155311 (2017).

[59] Y. Murotani and R. Shimano, Nonlinear optical response of collective modes in multiband superconductors assisted by nonmagnetic impurities, Phys. Rev. B 99, 224510 (2019).

[60] M. Silaev, Nonlinear electromagnetic response and Higgs-mode excitation in BCS superconductors with impurities, Phys. Rev. B 99, 224511 (2019).

[61] Y. Murotani, N. Tsuji, and H. Aoki, Theory of light-induced resonances with collective Higgs and Leggett modes in multiband superconductors, Phys. Rev. B 95, 104503 (2017).

[62] P. W. Anderson, Random-phase approximation in the theory of superconductivity, Phys. Rev. 112, 1900 (1958).

[63] J. R. Kirtley, C. C. Tsuei, Ariando, C. J. M. Verwijs, S. Harkema, and H. Hilgenkamp, Angle-resolved phase-sensitive determination of the in-plane gap symmetry in $\mathrm{YBa}_{2} \mathrm{Cu}_{3} \mathrm{O}_{7-\delta}$, Nat. Phys. 2, 190 (2006).

[64] M. Bakr, A. P. Schnyder, L. Klam, D. Manske, C. T. Lin, B. Keimer, M. Cardona, and C. Ulrich, Electronic and phononic Raman scattering in detwinned $\mathrm{YBa}_{2} \mathrm{Cu}_{3} \mathrm{O}_{6.95}$ and $\mathrm{Y}_{0.85} \mathrm{Ca}_{0.15} \mathrm{Ba}_{2} \mathrm{Cu}_{3} \mathrm{O}_{6.95}$ : $s$-wave admixture to the $d_{x^{2}-y^{2}}$-wave order parameter, Phys. Rev. B 80, 064505 (2009).

[65] A. P. Schnyder, C. Mudry, and D. Manske, Screening in $(d+$ $s$ )-wave superconductors: Application to Raman scattering, Phys. Rev. B 75, 174525 (2007).

[66] K. A. Müller, On the macroscopic s- and d-wave symmetry in cuprate superconductors, Philos. Mag. Lett. 82, 279 (2002). 